\title{
MATHEMATICAL STUDY OF A PETROLEUM-ENGINEERING SCHEME
}

\author{
Robert Eymard ${ }^{1}$, Raphà̀le Herbin ${ }^{2}$ And Anthony Michel ${ }^{3}$
}

\begin{abstract}
Models of two phase flows in porous media, used in petroleum engineering, lead to a system of two coupled equations with elliptic and parabolic degenerate terms, and two unknowns, the saturation and the pressure. For the purpose of their approximation, a coupled scheme, consisting in a finite volume method together with a phase-by-phase upstream weighting scheme, is used in the industrial setting. This paper presents a mathematical analysis of this coupled scheme, first showing that it satisfies some a priori estimates: the saturation is shown to remain in a fixed interval, and a discrete $L^{2}\left(0, T ; H^{1}(\Omega)\right)$ estimate is proved for both the pressure and a function of the saturation. Thanks to these properties, a subsequence of the sequence of approximate solutions is shown to converge to a weak solution of the continuous equations as the size of the discretization tends to zero.
\end{abstract}

Mathematics Subject Classification. 35K65, 76S05, 65M12.

Received October 8, 2002.

\section{INTRODUCTION}

Computing the flow of fluid chemical species in porous media takes an important place in oil recovery engineering. In several cases, the engineer should simultaneously represent the thermodynamical evolution of the hydrocarbon components during the pressure drop due to the extraction of oil, and the multi-phase flow (oil, water and gas) in the oil reservoir.

On the other hand, in the soil mechanics setting, engineers need to study the air-water flow in soils. They mainly used in the past the so-called Richards model, which has unfortunately been proven to be somewhat physically limited; thus, more and more engineers actually prefer the use of a two-phase flow model.

The importance of both applications has motivated number of works on multi-phase flows in porous media. The derivation of the mathematical equations describing this phenomenon may be found in [6,7]. A review of the models for oil reservoir engineering may also be found in $[10,31]$. The mathematical analysis of the resulting equations (with varying assumptions) has been developed for some time now, see e.g. $[1-3,9,10,12-14,25-27,30,32,33]$. Here, as in most of these references, we shall deal with the simplified case where the two fluids are assumed to be incompressible and immiscible (the petroleum engineering "deadoil" model). Let us furthermore assume that the reservoir is a horizontal homogeneous isotropic domain (thus leading to the disappearance of gravity terms). In the absence of a volumetric source term, the conservation

\footnotetext{
Keywords and phrases. Multiphase flow, Darcy's law, porous media, finite volume scheme.

${ }^{1}$ Université de Marne-la-Vallée, 5 Bld Descartes, Bat. Lavoisier, 77454 Marne-la-Vallée, France. e-mail: eymard@math.univ-mlv.fr

2 Université de Aix-Marseille 1, 39 rue Joliot Curie, 13453 Marseille, France. e-mail: herbin@cmi.univ-mrs.fr

3 Institut Français du Pétrole, 1 et 4 avenue Bois Préau, 92000 Rueil-Malmaison, France. e-mail: anthony.michel@ifp.fr
} 
equations for such a two-phase flow in this particular case, using Darcy's law, may be written as:

$$
\begin{aligned}
u_{t}-\operatorname{div}\left(k_{1}(u) \nabla p\right) & =0, \\
(1-u)_{t}-\operatorname{div}\left(k_{2}(u) \nabla q\right) & =0, \\
q-p & =p_{c}(u),
\end{aligned}
$$

where $u$ and $p$ are respectively the saturation and the pressure of the wetting fluid (the other fluid is called the non-wetting fluid), $k_{1}$ and $k_{2}$ are respectively the mobilities of the wetting fluid and the mobility of the non-wetting fluid and $p_{c}$ is the capillary pressure. In system (1.1), the physical functions $k_{1}, k_{2}$ and $p_{c}$ are supposed to only depend on the saturation $u$ of the wetting fluid (in more realistic heterogeneous cases, these functions should also depend on the rock type).

The numerical discretization of the above equations has been the object of several studies during the past decades. The description of the numerical treatment by finite differences may be found in the books by Peaceman [36] and Aziz and Settari [5]. Mixed or hybrid finite element methods were also extensively studied in the past years see $e . g .[4,10,18,19]$; they have the advantage of an amenable mathematical setting. Their use with a lagrangian-eulerian formulation for the treatment of the convection term was also studied [39]. However, finite volume type methods are often preferred in actual computational codes because they are cheap (with respect to the programming and computational times), and because they allow to define the discrete unknowns at the same location. This last property is important when dealing with complex thermodynamics (in reservoir engineering for instance), where algebraic equations between the discrete unknowns must be taken into account. Among the finite volume methods for two-phase flow in porous media one may cite the control volume finite element method $[28,29]$ or the cell-centered finite volume, an introduction to which can be found in [24]. A proof of convergence of these two schemes to a weak solution of system (1.1) in the case $k_{1}(u)=u, k_{2}(u)=1-u$ and $p_{c}=0$ is given in [20] for the control volume finite element scheme, and in [38] (see also [24]) for the cell-centered finite volume scheme. Let us also mention the earlier works $[8,37]$ on the convergence of a "phase by phase" upstream weighting cell-centered finite volume in the one dimensional case, with $p_{c}=0$ and in presence of gravity terms.

In the case of more general functions $k_{1}, k_{2}$ and $p_{c}$, the convergence of a cell-centered finite volume scheme to a weak solution of system (1.1) is studied in [34]: system (1.1) is first rewritten as

$$
\begin{aligned}
u_{t}+\operatorname{div}\left(f_{1}(u) F\right)-\Delta g(u) & =0, \\
(1-u)_{t}+\operatorname{div}\left(\left(1-f_{1}(u)\right) F\right)+\Delta g(u) & =0, \\
F+\left(k_{1}(u)+k_{2}(u)\right) \nabla p+k_{2}(u) \nabla p_{c}(u) & =0, \\
f_{1}(u)=k_{1}(u) /\left(k_{1}(u)+k_{2}(u)\right) & \\
g^{\prime}(u)=-\left(k_{1}(u) k_{2}(u) p_{c}{ }^{\prime}(u)\right) /\left(k_{1}(u)+k_{2}(u)\right) &
\end{aligned}
$$

and the cell-centered finite volume scheme studied in [34] consists in a centered finite difference scheme for (1.4), and an upstream weighting scheme for $f_{1}(u)$ in $(1.2,1.3)$ coupled with a finite difference scheme for the evaluation of $\nabla g(u)$. Although this scheme could be generalized to more realistic physical cases, a cell-centered finite volume scheme, written on the original nonlinear system (1.1), using a "phase by phase" upstream choice for computations of the fluxes (namely scheme (3.22)-(3.26) presented below) is preferred in the industrial setting. It seems that at least two reasons can explain this preference: the scheme (3.22)-(3.26) appears to be easier to implement and more robust [35]. However, its mathematical analysis is more difficult because of the upwinding error terms, as we shall see below .

The aim of the present paper is to show that the approximate solution obtained with the finite volume scheme (3.22)-(3.26) converges, as the mesh size tends to zero, to a solution of system (1.1) in an appropriate sense defined in Section 2. In Section 3 we introduce the finite volume discretization, the numerical scheme and state the main convergence results. The remainder of the paper is devoted to the proof of this result: in Section 4, a priori estimates on the approximate solution are derived; in Section 5 we prove the compactness of 
sequences of approximate solutions. The passage to the limit on the scheme, performed in Section 6 concludes the proof of convergence and some numerical results are stated in Section 7. We end this paper with some concluding remarks on open problems.

\section{Mathematical formulation of the Continuous Problem}

We now give a more complete formulation to system (1.1). Let $\Omega$ be an open bounded subset of $\mathbb{R}^{d}(d \geq 0)$, let $T \in \mathbb{R}_{+}$. The saturation $u: \Omega \times(0, T) \rightarrow \mathbb{R}$ and the pressure $p: \Omega \times(0, T) \rightarrow \mathbb{R}$ of the wetting fluid are solution to the following coupled system:

$$
\begin{aligned}
u_{t}-\operatorname{div}\left(k_{1}(u) \nabla p\right) & =f_{1}(c) \bar{s}-f_{1}(u) \underline{s} \text { on } \Omega \times(0, T), \\
(1-u)_{t}-\operatorname{div}\left(k_{2}(u) \nabla q\right) & =f_{2}(c) \bar{s}-f_{2}(u) \underline{s} \text { on } \Omega \times(0, T), \\
q-p & =p_{c}(u),
\end{aligned}
$$

with the following Neumann boundary conditions:

$$
\begin{array}{ll}
\nabla p \cdot \mathbf{n}=0 & \text { on } \partial \Omega \times(0, T), \\
\nabla q \cdot \mathbf{n}=0 & \text { on } \partial \Omega \times(0, T),
\end{array}
$$

the following initial condition:

$$
u(\cdot, 0)=u_{0} \text { on } \Omega
$$

and, since both fluids are incompressible, we prescribe the following arbitrary condition on $p$

$$
\int_{\Omega} p(x, \cdot) \mathrm{d} x=0 \text { on }(0, T) .
$$

We recall that the functions $k_{1}(u)$ and $k_{2}(u)$ respectively denote the mobilities of the wetting fluid and of the non-wetting fluid and the function $p_{c}(u)$ represents the capillary pressure. The functions $\bar{s}$ and $\underline{s}$ stand respectively for an injection and a production volumetric flow rate. The composition of the injected fluid in the wetting and non-wetting components is prescribed by the imposed input saturation $c$, whereas that of the produced fluid depends on the saturation $u$, by the way of the function $f_{1}$ which is called "the fractional flow" of the wetting phase i.e.:

$$
f_{1}(a)=\frac{k_{1}(a)}{k_{1}(a)+k_{2}(a)}, \forall a \in[0,1] .
$$

Similarly, we denote by $f_{2}$ the fractional flow of the non-wetting phase, i.e.:

$$
f_{2}(a)=\frac{k_{2}(a)}{k_{1}(a)+k_{2}(a)}=1-f_{1}(a), \forall a \in[0,1] .
$$

The data is assumed to satisfy the following assumptions:

$\Omega$ is a polygonal connected subset of $\mathbb{R}^{d}, d=2$ or $3, T>0$ is given,

$$
u_{0} \in L^{\infty}(\Omega) \text { and } 0 \leq u_{0} \leq 1 \text { a.e. in } \Omega,
$$

$$
c \in L^{\infty}(\Omega \times(0, T)) \text { and } 0 \leq c \leq 1 \text { a.e. in } \Omega \times(0, T),
$$




$$
\left\{\begin{array}{l}
\bar{s}, \underline{s} \in L^{2}(\Omega \times(0, T)), \bar{s} \text { and } \underline{s} \geq 0 \text { a.e. } \\
\text { and } \int_{\Omega \times(0, T)}(\bar{s}(x, t)-\underline{s}(x, t)) \mathrm{d} x \mathrm{~d} t=0 .
\end{array}\right.
$$

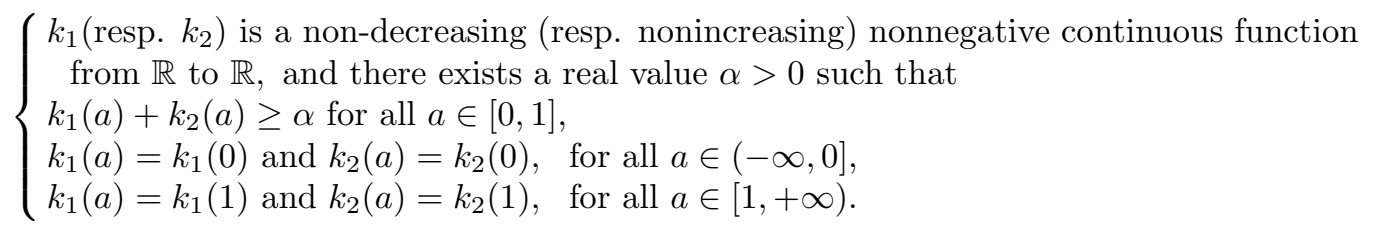

$$
\left\{\begin{array}{l}
p_{c} \text { is a nonincreasing continuous function from } \mathbb{R} \text { to } \mathbb{R} \\
\text { such that } p_{c \mid(0,1)}^{\prime} \in L^{1}(0,1), \\
p_{c}(a)=p_{c}(0), \text { for all } a \in(-\infty, 0], p_{c}(a)=p_{c}(1), \text { for all } a \in[1,+\infty) .
\end{array}\right.
$$

We show in Figure 1 a typical behaviour of the functions $k_{1}, k_{2}$ (relative mobilities), $f_{1}$ (fractional flow), $k_{1}+k_{2}$ (total mobility) and $p_{c}$ (capillary pressure).

Remark 2.1. The functions $k_{1}, k_{2}$ and $p_{c}$ are defined on $\mathbb{R}$, and not only on $[0,1]$, in order to ensure the discrete maximum principle and thus the existence of a physically admissible discrete solution (see Sect. 4).

Following Chavent [10], in order to obtain a weak formulation (which will be shown to be the limit of the numerical scheme), we introduce some artificial pressures, which are however not actually used in the implementation of the scheme. These artificial pressures are denoted by $p_{g}$ and $q_{g}$ and defined by:

$$
p_{g}(b)=\int_{0}^{b} \frac{k_{2}(a)}{k_{1}(a)+k_{2}(a)} p_{c}{ }^{\prime}(a) \mathrm{d} a \text { and } q_{g}(b)=\int_{0}^{b} \frac{k_{1}(a)}{k_{1}(a)+k_{2}(a)} p_{c}{ }^{\prime}(a) \mathrm{d} a, \forall b \in[0,1]
$$

(note that $p+p_{g}(u)=q-q_{g}(u)$ because of the condition $q-p=p_{c}(u)$ ). Let us finally define the function $g$ from $[0,1]$ to $\mathbb{R}$ by:

$$
g(b)=-\int_{0}^{b} \frac{k_{1}(a) k_{2}(a)}{k_{1}(a)+k_{2}(a)} p_{c}^{\prime}(a) \mathrm{d} a, \forall b \in[0,1] .
$$

Definition 2.1 (Weak solution). Under assumptions and Definitions (2.12)-(2.21), the pair $(u, p)$ is a weak solution of problem (2.5)-(2.11) if

$$
\begin{aligned}
& u \in L^{\infty}(\Omega \times(0, T)), \text { with } 0 \leq u(x, t) \leq 1 \text { for a.e. }(x, t) \in \Omega \times(0, T), \\
& p \in L^{2}(\Omega \times(0, T)), \\
& p+p_{g}(u) \in L^{2}\left(0, T ; H^{1}(\Omega)\right), \\
& g(u) \in L^{2}\left(0, T ; H^{1}(\Omega)\right),
\end{aligned}
$$

and for every function $\varphi \in C^{\infty}\left(\mathbb{R}^{d} \times \mathbb{R}\right)$ such that $\varphi(\cdot, T)=0$,

$$
\begin{aligned}
\int_{0}^{T} \int_{\Omega}\left[u(x, t) \varphi_{t}(x, t)-\left(k_{1}(u(x, t)) \nabla\left(p+p_{g}(u)\right)(x, t)-\nabla g(u(x, t))\right) \cdot \nabla \varphi(x, t)\right] \mathrm{d} x \mathrm{~d} t & \\
& \quad+\int_{0}^{T} \int_{\Omega}\left[f_{1}(c(x, t)) \bar{s}(x, t)-f_{1}(u(x, t)) \underline{s}(x, t)\right] \varphi(x, t) \mathrm{d} x \mathrm{~d} t+\int_{\Omega} u_{0}(x) \varphi(x, 0) \mathrm{d} x=0,
\end{aligned}
$$



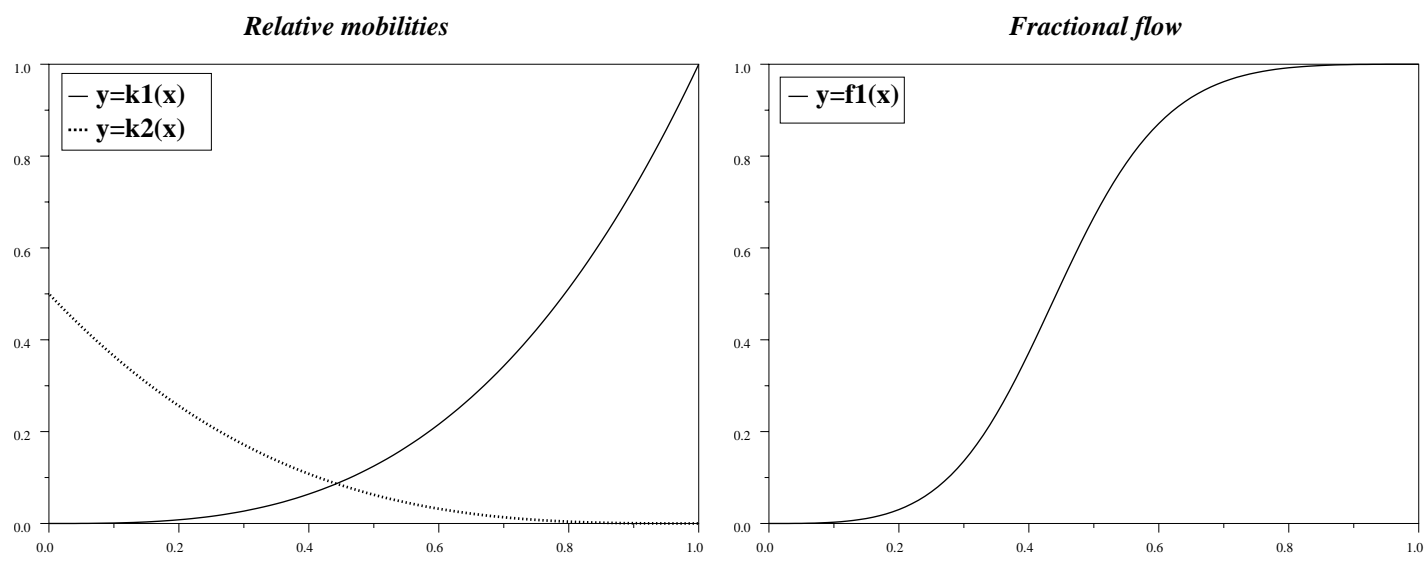

Capillary pressure

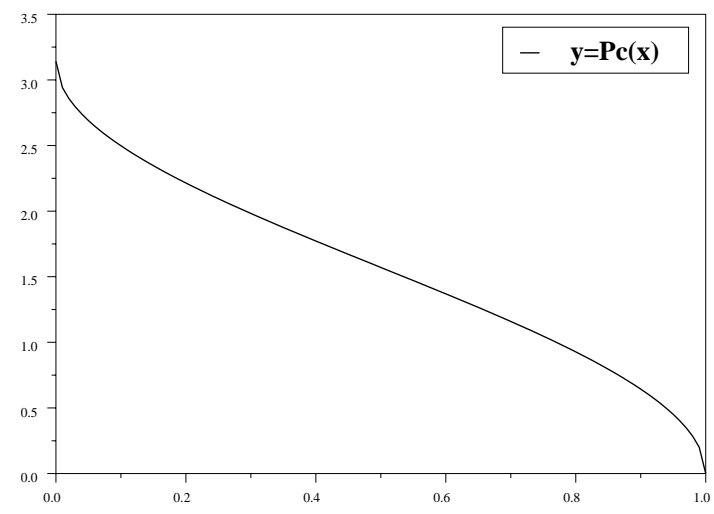

Figure 1. Behaviour of the functions $k_{1}, k_{2}, f_{1}, p_{c}$.

$$
\begin{gathered}
\int_{0}^{T} \int_{\Omega}\left[(1-u(x, t)) \varphi_{t}(x, t)-\left(k_{2}(u(x, t)) \nabla\left(p+p_{g}(u)\right)(x, t)+\nabla g(u(x, t))\right) \cdot \nabla \varphi(x, t)\right] \mathrm{d} x \mathrm{~d} t \\
+\int_{0}^{T} \int_{\Omega}\left[f_{2}(c(x, t)) \bar{s}(x, t)-f_{2}(u(x, t)) \underline{s}(x, t)\right] \varphi(x, t) \mathrm{d} x \mathrm{~d} t+\int_{\Omega}\left(1-u_{0}(x)\right) \varphi(x, 0) \mathrm{d} x=0 \\
\int_{\Omega} p(x, t) \mathrm{d} x=0 \text { for a.e. } t \in(0, T) .
\end{gathered}
$$

Remark 2.2. One may remark that in the above formulation, the fact that $p \in L^{2}(\Omega \times(0, T))$ is implied by the fact that $p+p_{g}(u) \in L^{2}\left(0, T ; H^{1}(\Omega)\right)$ and $p_{g}(u)$ is bounded on $\Omega \times(0, T)$. Also note that the terms $\left.k_{1}(u)\right) \nabla(p+$ $\left.p_{g}(u)\right)-\nabla g(u)$ (resp. $\left.k_{2}(u) \nabla\left(p+p_{g}(u)\right)+\nabla g(u)\right)$ are formally equal to $k_{1}(u) \nabla p\left(\operatorname{resp} . \quad k_{2}(u) \nabla\left(p+p_{c}(u)\right)\right)$. However, these last two terms are not properly defined under the regularity assumptions of the above definition.

The existence of a weak solution $(u, p)$ to $(2.5)-(2.11)$ in the sense of Definition 2.1 will be obtained as a by-product of the convergence of the numerical scheme. Note that existence was also shown in [11] for a system taking gravity into account. To our knowledge, no uniqueness result is known under the generic assumptions of Theorem 3.1 below. In $[11,12]$, uniqueness is proven under a condition which corresponds, roughly speaking, to the assumption that the inequality $\left[\left(k_{1}+k_{2}\right)^{\prime}(s)\right]^{2} \leq-C p_{c}^{\prime}(s) \frac{k_{1}(s) k_{2}(s)}{k_{1}(s)+k_{2}(s)}$ holds for any $s \in[0,1]^{2}$. Note that this condition excludes, for instance, the simple case where $k_{1}$ or $k_{2}$ are convex functions (see Fig. 1). 


\section{The Finite Volume scheme}

\subsection{Finite volume definitions and notations}

Following [24], let us define a finite volume discretization of $\Omega \times(0, T)$.

Definition 3.1 (Admissible mesh of $\Omega$ ). An admissible mesh $\mathcal{T}$ of $\Omega$ is given by a set of open bounded polygonal convex subsets of $\Omega$ called control volumes and a family of points (the "centers" of control volumes) satisfying the following properties:

(1) The closure of the union of all control volumes is $\bar{\Omega}$. We denote by $m_{K}$ the measure of $K$, and define

$$
\operatorname{size}(\mathcal{T})=\max \{\operatorname{diam}(K), K \in \mathcal{T}\}
$$

(2) For any $(K, L) \in \mathcal{T}^{2}$ with $K \neq L$, then $K \cap L=\emptyset$. One denotes by $\mathcal{E} \subset \mathcal{T}^{2}$ the set of $(K, L)$ such that the $d$-1-Lebesgue measure of $\bar{K} \cap \bar{L}$ is positive. For $(K, L) \in \mathcal{E}$, one denotes $K \mid L=\bar{K} \cap \bar{L}$ and $m_{K \mid L}$ the $d$-1-Lebesgue measure of $K \mid L$.

(3) For any $K \in \mathcal{T}$, one defines $\mathcal{N}_{K}=\{L \in \mathcal{T},(K, L) \in \mathcal{E}\}$ and one assumes that $\partial K=\bar{K} \backslash K=$ $(\bar{K} \cap \partial \Omega) \cup \bigcup_{L \in \mathcal{N}_{K}} K \mid L$.

(4) The family of points $\left(x_{K}\right)_{K \in \mathcal{T}}$ is such that $x_{K} \in K$ (for all $K \in \mathcal{T}$ ) and, if $L \in \mathcal{N}_{K}$, it is assumed that the straight line $\left(x_{K}, x_{L}\right)$ is orthogonal to $K \mid L$. We set $d_{K \mid L}=d\left(x_{K}, x_{L}\right)$ and $\tau_{K \mid L}=\frac{m_{K \mid L}}{d_{K \mid L}}$, that is sometimes called the "transmissivity" through $K \mid L$.

The problem under consideration is time-dependent, hence we also need to discretize the time interval $(0, T)$.

Definition 3.2 (Time discretization). A time discretization of $(0, T)$ is given by an integer value $N$ and by a strictly increasing sequence of real values $\left(t^{n}\right)_{n \in \llbracket 0, N+1 \rrbracket}$ with $t^{0}=0$ and $t^{N+1}=T$. The time steps are then defined by $\delta t^{n}=t^{n+1}-t^{n}$, for $n \in \llbracket 0, N \rrbracket$.

We may then define a discretization of the whole domain $\Omega \times(0, T)$ in the following way:

Definition 3.3 (Discretization of $\Omega \times(0, T)$ ). A finite volume discretization $D$ of $\Omega \times(0, T)$ is defined by

$$
D=\left(\mathcal{T}, \mathcal{E},\left(x_{K}\right)_{K \in \mathcal{T}}, N,\left(t^{n}\right)_{n \in \llbracket 0, N \rrbracket}\right)
$$

where $\mathcal{T}, \mathcal{E},\left(x_{K}\right)_{K \in \mathcal{T}}$ is an admissible mesh of $\Omega$ in the sense of Definition 3.1 and $N,\left(t^{n}\right)_{n \in \llbracket 0, N+1 \rrbracket}$ is a time discretization of $(0, T)$ in the sense of Definition 3.2. One then sets

$$
\operatorname{size}(D)=\max \left(\operatorname{size}(\mathcal{T}),\left(\delta t^{n}\right)_{n \in \llbracket 0, N \rrbracket} .\right.
$$

Definition 3.4 (Discrete functions and notations). Let $D$ be a discretization of $\Omega \times(0, T)$ in the sense of Definition 3.3. We use a capital letter with the subscript $D$ to denote any function from $\mathcal{T} \times \llbracket 0, N+1 \rrbracket$ to $\mathbb{R}$ ( $U_{D}$ or $P_{D}$ for instance) and we denote its value at the point $(K, n)$ using the subscript $K$ and the superscript $n$ $\left(U_{K}^{n}\right.$ for instance, we then denote $\left.U_{D}=\left(U_{K}^{n}\right)_{K \in \mathcal{T}, n \in \llbracket 0, N+1 \rrbracket}\right)$. To any discrete function $U_{D}$ corresponds an approximate function $u_{D}$ defined almost everywhere on $\Omega \times(0, T)$ by:

$$
u_{D}(x, t)=U_{K}^{n+1} \text {, for a.e. }(x, t) \in K \times\left(t^{n}, t^{n+1}\right), \forall K \in \mathcal{T}, \forall n \in \llbracket 0, N \rrbracket \text {. }
$$

For any continuous function $f: \mathbb{R} \mapsto \mathbb{R}, f\left(U_{D}\right)$ denotes the discrete function $(K, n) \mapsto f\left(U_{K}^{n+1}\right)$. If $L \in \mathcal{N}_{K}$, and $U_{D}$ is a discrete function, we denote by $\delta_{K, L}^{n+1}(U)=U_{L}^{n+1}-U_{K}^{n+1}$. For example, $\delta_{K, L}^{n+1}\left(f_{1}(U)\right)=f_{1}\left(U_{L}^{n+1}\right)-$ $f_{1}\left(U_{K}^{n+1}\right)$. 


\subsection{The coupled finite volume scheme}

The finite volume scheme is obtained by writing the balance equations of the fluxes on each control volume. Let $D$ be a discretization of $\Omega \times(0, T)$ in the sense of Definition 3.3. Let us integrate equations (2.5, 2.6) over each control volume $K$. By using the Green-Riemann formula, if $\Phi$ is a vector field, the integral of $\operatorname{div}(\Phi)$ on a control volume $K$ is equal to the sum of the normal fluxes of $\Phi$ on the edges. Here we apply this formula to $\Phi_{1}=k_{1}(u) \nabla p$ and $\Phi_{2}=k_{2}(u) \nabla\left(p+p_{c}(u)\right)$. The resulting equation is discretized with a time implicit finite difference scheme; the normal gradients are discretized with a centered finite difference scheme. If we denote by $U_{D}=\left(U_{K}^{n}\right)_{K \in \mathcal{T}, n \in \llbracket 0, N+1 \rrbracket}$ and $P_{D}=\left(P_{K}^{n}\right)_{K \in \mathcal{T}, n \in \llbracket 1, N+1 \rrbracket}$ the discrete unknowns corresponding to $u$ and $p$, the finite volume scheme that we obtain is the following set of equations:

$$
\begin{aligned}
U_{K}^{0}=\frac{1}{m_{K}} \int_{K} u_{0}(x) \mathrm{d} x, & \text { for all } K \in \mathcal{T}, \\
\frac{U_{K}^{n+1}-U_{K}^{n}}{\delta t^{n}} m_{K}-\sum_{L \in \mathcal{N}_{K}} \tau_{K \mid L} k_{1, K \mid L}^{n+1} \delta_{K, L}^{n+1}(P) & =m_{K}\left(f_{1}\left(c_{K}^{n+1}\right) \bar{s}_{K}^{n+1}-f_{1}\left(U_{K}^{n+1}\right) \underline{s}_{K}^{n+1}\right), \\
\frac{\left(1-U_{K}^{n+1}\right)-\left(1-U_{K}^{n}\right)}{\delta t^{n}} m_{K}-\sum_{L \in \mathcal{N}_{K}} \tau_{K \mid L} k_{2, K \mid L}^{n+1} \delta_{K, L}^{n+1}(Q) & =m_{K}\left(f_{2}\left(c_{K}^{n+1}\right) \bar{s}_{K}^{n+1}-f_{2}\left(U_{K}^{n+1}\right) \underline{s}_{K}^{n+1}\right), \\
Q_{K}^{n+1}-P_{K}^{n+1} & =p_{c}\left(U_{K}^{n+1}\right),
\end{aligned}
$$

for all $(K, n) \in \mathcal{T} \times \llbracket 0, N \rrbracket$, and

$$
\sum_{K \in \mathcal{T}} m_{K} P_{K}^{n+1}=0, \text { for all } n \in \llbracket 0, N \rrbracket,
$$

where

- $c_{K}^{n+1}$ is the mean value of $c$ over the time-space cell $K \times\left(t^{n}, t^{n+1}\right)$,

- $\bar{s}_{K}^{n+1}$ and $\underline{s}_{K}^{n+1}$ denote the mean values of $\bar{s}$ and $\underline{s}$ over the time-space cell $K \times\left(t^{n}, t^{n+1}\right)$,

- $k_{1, K \mid L}^{n+1}$ and $k_{2, K \mid L}^{n+1}$ denote the upwind discretization of $k_{1}(u)$ (or $k_{2}(u)$ ) on the interface $K \mid L$, which are defined by:

$$
k_{1, K \mid L}^{n+1}=k_{1}\left(U_{1, K \mid L}^{n+1}\right) \text { and } k_{2, K \mid L}^{n+1}=k_{2}\left(U_{2, K \mid L}^{n+1}\right)
$$

with

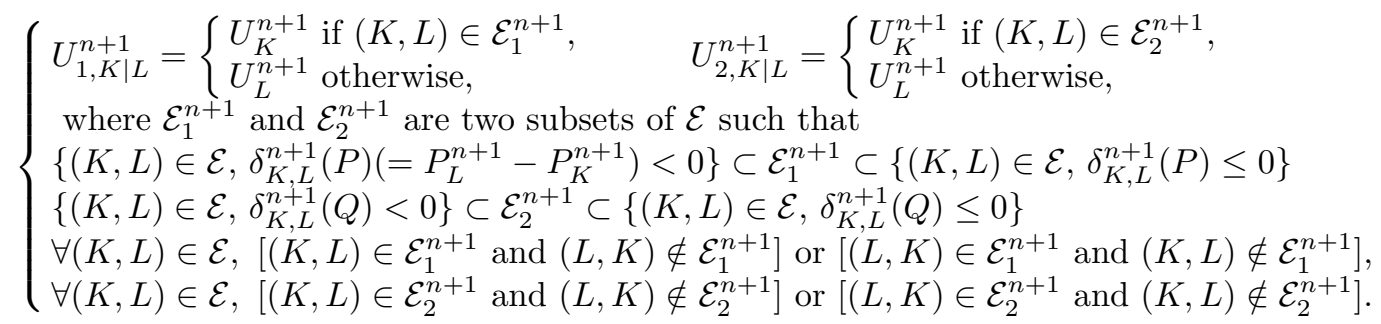

Remark 3.1. The formulae (3.28) express a phase by phase upstream choice: the value of the mobility of each phase on the edge $(K, L)$ is determined by the sign of the difference of the discrete pressure. Note that, for all $(K, L) \in \mathcal{E}$ and $n \in \llbracket 0, N \rrbracket$ such that $P_{K}^{n+1}=P_{L}^{n+1}$, the choice between $(K, L) \in \mathcal{E}_{1}^{n+1}$ and $(L, K) \in \mathcal{E}_{1}^{n+1}$ can be arbitrarily done, without modifying the equation (3.23) (the same remark holds for the set $\mathcal{E}_{2}^{n+1}$, the equation (3.24) and all the pairs $(K, L) \in \mathcal{E}$ and $n \in \llbracket 0, N \rrbracket$ such that $\left.Q_{K}^{n+1}=Q_{L}^{n+1}\right)$. Thus, the scheme $(3.22)-(3.26)$ does not depend on the choice of the pair of subsets $\left(\mathcal{E}_{1}^{n+1}, \mathcal{E}_{2}^{n+1}\right)$ satisfying (3.28) when there are more than one such pair (there exists at least one). 
We show below (see Prop. 4.3) that there exists at least one solution to this scheme. From this discrete solution, we build an approximate solution $\left(u_{D}, p_{D}\right)$ defined almost everywhere on $\Omega \times(0, T)$ by (see Def. 3.4):

$$
\begin{aligned}
& u_{D}(x, t)=U_{K}^{n+1}, \quad \forall x \in K, \forall t \in\left(t^{n}, t^{n+1}\right), \\
& p_{D}(x, t)=P_{K}^{n+1}, \quad \forall x \in K, \forall t \in\left(t^{n}, t^{n+1}\right) .
\end{aligned}
$$

Remark 3.2. The discretization scheme yields a nonlinear system of equations which is solved in practice by the Newton method. Numerical experiments show that if the time step is adequately chosen, the Newton procedure converges with a small number of iterations. Hence, although it is implicit, this scheme is cheaper than the analogous explicit one, since (disregarding the problem of accuracy) the time step may be taken much larger than the explicit time step given by the CFL condition.

We may now state the main convergence result.

Theorem 3.1. Under assumptions (2.14)-(2.19), let us furthermore assume that:

$$
\begin{aligned}
& \left\{\begin{array}{l}
k_{1}(0)=k_{2}(1)=0 \text { and, for } i=1,2, k_{i}^{\prime} \in L^{\infty}(0,1) \text { and there exist } \theta_{i} \geq 1 \text { and } \alpha_{i}>0 \\
\text { such that } \alpha_{1} b^{\theta_{1}-1} \leq k_{1}^{\prime}(b) \leq \frac{1}{\alpha_{1}} b^{\theta_{1}-1} \text { and } \\
\alpha_{2}(1-b)^{\theta_{2}-1} \leq-k_{2}^{\prime}(b) \leq \frac{1}{\alpha_{2}}(1-b)^{\theta_{2}-1}, \text { for a.e. } b \in(0,1),
\end{array}\right. \\
& \left\{\begin{array}{l}
\text { there exist } \alpha_{0}>0, \beta_{0}>0, \beta_{1}>0, \text { such that } \\
\frac{1}{\alpha_{0}} b^{\beta_{0}-1}(1-b)^{\beta_{1}-1} \geq-p_{c}{ }^{\prime}(b) \geq \alpha_{0} b^{\beta_{0}-1}(1-b)^{\beta_{1}-1}, \text { for a.e. } b \in(0,1) .
\end{array}\right.
\end{aligned}
$$

Let $\left(D_{m}\right)_{m \in \mathbb{N}}$ be a sequence of discretizations of $\Omega \times(0, T)$ in the sense of Definition 3.3 such that $\lim _{m \rightarrow \infty} \operatorname{size}\left(D_{m}\right)=$ 0 and satisfying the following uniform regularity property:

$$
\exists \theta \in \mathbb{R}_{+} \text {such that } \forall K \in \mathcal{T}_{m}, \sum_{L \in \mathcal{N}_{K}} m_{K \mid L} d_{K \mid L} \leq \theta m_{K} .
$$

Let $\left(u_{D_{m}}, p_{D_{m}}\right)_{m \in \mathbb{N}}$ be a sequence of approximate solutions defined by (3.29) and the finite volume scheme (3.22)-(3.28) for the sequence of discretizations $\left(D_{m}\right)_{m \in \mathbb{N}}$. Then there exists a subsequence of $\left(u_{D_{m}}, p_{D_{m}}\right)_{m \in \mathbb{N}}$, still denoted $\left(u_{D_{m}}, p_{D_{m}}\right)_{m \in \mathbb{N}}$, and a weak solution $(u, p)$ of (2.5)-(2.11) such that $u_{D_{m}}$ tends to $u$ in $L^{r}(\Omega \times(0, T))$ for all $r \in[1,+\infty)$ and $p_{D_{m}}$ tends to $p$ weakly in $L^{2}(\Omega \times(0, T))$, as $m$ tends to infinity.

Proof. Let $\left(D_{m}\right)_{m \in \mathbb{N}}$ be a sequence of admissible discretizations $\left(D_{m}\right)_{m \in \mathbb{N}}$ such that $\lim _{m \rightarrow+\infty} \operatorname{size}\left(D_{m}\right)=0$. Thanks to Proposition 4.3, of Section 4 below, there exists a sequence of approximate solutions $\left(u_{D_{m}}, p_{D_{m}}\right)_{m \in \mathbb{N}}$ given by the finite volume scheme (3.22)-(3.28) for the sequence of discretizations $\left(D_{m}\right)_{m \in \mathbb{N}}$. Thanks to the $L^{\infty}$ estimate which is established in Proposition 4.1 of Section 4, and to the compactness properties given in Corollary 5.2 and Proposition 5.1 of Section 5, we find that the sequence $\left(g\left(u_{D_{m}}\right)\right)_{m \in \mathbb{N}}$ satisfies the hypotheses of Corollary 9.1 of Kolmogorov's theorem (this corollary is given in the appendix). Hence there exists a function $\tilde{g} \in L^{2}(\Omega \times(0, T))$ such that, up to a subsequence, $g\left(u_{D_{m}}\right)$ tends to $\tilde{g}$ in $L^{2}(\Omega \times(0, T))$ as $m$ tends to infinity. Passing to the limit in (4.64) of Corollary 4.1 as $m$ tends to infinity, we get that $\tilde{g} \in L^{2}\left(0, T ; H^{1}(\Omega)\right)$. Since $g$ is increasing and $u_{D_{m}}$ remains bounded, it follows that $u_{D_{m}}$ tends to $u:=g^{-1}(\tilde{g})$ in $L^{r}(\Omega \times(0, T))$ for all $r \in[1,+\infty)$, and that $p_{g}\left(u_{D_{m}}\right)$ tends to $p_{g}(u)$ in $L^{r}(\Omega \times(0, T))$ for all $r \in[1,+\infty)$, as $m$ tends to infinity.

Let us then remark that $\int_{\Omega} p_{D_{m}}(x) \mathrm{d} x=0$, that $\int_{\Omega} p_{g}\left(u_{D_{m}}\right)(x) \mathrm{d} x$ is bounded. Hence since $\Omega$ is connected, we may use the discrete Poincaré-Wirtinger inequality [24] to obtain from the discrete $H^{1}$ estimate (4.45) given in 
Proposition 4.2 that $p_{D_{m}}+p_{g}\left(u_{D_{m}}\right)$ remains bounded in $L^{2}(\Omega \times(0, T))$; therefore there exists $\tilde{p} \in L^{2}(\Omega \times(0, T))$ such that $p_{D_{m}}+p_{g}\left(u_{D_{m}}\right)$ tends to $\tilde{p}$ weakly in $L^{2}(\Omega \times(0, T))$ as $m$ tends to infinity. Thanks to the estimate on the translates of the pressure (5.65) given in Proposition 5.1 (in Sect. 5) we then obtain, using regular test functions $\varphi$, that $\int_{\Omega \times(0, T)} \tilde{p}(x, t) \partial \varphi_{i}(x, t) \mathrm{d} x \mathrm{~d} t \leq C_{1}\|\varphi\|_{L^{2}(\Omega \times(0, T))}$. Hence $\tilde{p} \in L^{2}\left(0, T ; H^{1}(\Omega)\right)$. It follows that $p_{D_{m}}$ tends to $p:=\tilde{p}-p_{g}(u) \in L^{2}(\Omega \times(0, T))$ weakly in $L^{2}(\Omega \times(0, T))$ as $m$ tends to infinity. In order to conclude the proof of Theorem 3.1, there only remains to prove that $(u, p)$ is a weak solution of problem (2.5)-(2.11) in the sense of Definition 2.1. This is a direct consequence of Theorem 6.1 given in Section 6.

Remark 3.3. If uniqueness of the weak solution holds, then of course, by a classical argument, the whole sequence $\left(u_{D_{m}}, p_{D_{m}}\right)_{m \in \mathbb{N}}$ of approximate solutions can be shown to converge to the weak solution. An error estimate might then also be obtained. However, as we earlier mentioned, uniqueness of the solution under the present assumptions is still an open problem.

\section{A priori estimates And existence of the Approximate solution}

In this section, we develop the first part of the proof of Theorem 3.1. The method used to prove these estimates is quite different from the one used in the previous related papers [23,34]. Note that all of these estimates hold even in the strongly degenerate case, that is if $p_{c}{ }^{\prime}=0$ on a nonempty open subset of $(0,1)$ (no other assumption than (2.19) is needed on the capillary pressure), except Corollary 4.1. However, this corollary is essential for the convergence Theorem 3.1.

\subsection{The maximum principle}

Let us show here that the phase by phase upstream choice yields the $L^{\infty}$ stability of the scheme.

Proposition 4.1 (Maximum principle). Under assumptions and notations (2.12)-(2.21), we denote by $u_{\min }, u_{\max } \in[0,1]$ some real values such that $u_{\min } \leq u_{0} \leq u_{\max }$ a.e. in $\Omega$ and $u_{\min } \leq c \leq u_{\max }$ a.e. in $\Omega \times(0, T)$. Let $D=\left(\mathcal{T}, \mathcal{E},\left(x_{K}\right)_{K \in \mathcal{T}}, N,\left(t^{n}\right)_{n \in \llbracket 0, N \rrbracket}\right)$ be a discretization of $\Omega \times(0, T)$ in the sense of Definition 3.3 and assume that $\left(U_{D}, P_{D}\right)$ is a solution of the finite volume scheme (3.22)-(3.26). Then the following maximum principle holds:

$$
u_{\min } \leq U_{K}^{n} \leq u_{\max }, \forall K \in \mathcal{T}, \forall n \in \llbracket 0, N+1 \rrbracket .
$$

Proof. By symmetry, we only need to prove the right part of inequality (4.33). By contradiction, let us assume that the maximum value of $U_{D}$ on $\mathcal{T} \times \llbracket 0, N+1 \rrbracket$ is larger than $u_{\max }$. Then this maximum value cannot be attained for $U_{K}^{0}$, since the initial condition (3.22) clearly implies that $U_{K}^{0} \leq u_{\max }$. Hence there exists $n \geq 0$ such that the maximum value of $U_{D}$ is $U_{K}^{n+1}$. If $n$ is chosen minimal, $U_{K}^{n+1}>U_{K}^{n}$ so by using (3.23) and (3.24) we have:

$$
\begin{array}{r}
\sum_{L \in \mathcal{N}_{K}} \tau_{K \mid L} k_{1, K \mid L}^{n+1} \delta_{K, L}^{n+1}(P)+m_{K}\left(f_{1}\left(c_{K}^{n+1}\right) \bar{s}_{K}^{n+1}-f_{1}\left(U_{K}^{n+1}\right) \underline{s}_{K}^{n+1}\right)>0, \\
-\sum_{L \in \mathcal{N}_{K}} \tau_{K \mid L} k_{2, K \mid L}^{n+1} \delta_{K, L}^{n+1}(Q)-m_{K}\left(f_{2}\left(c_{K}^{n+1}\right) \bar{s}_{K}^{n+1}-f_{2}\left(U_{K}^{n+1}\right) \underline{s}_{K}^{n+1}\right)>0 .
\end{array}
$$

By definition of the upwind approximation (3.27), the terms $\tau_{K \mid L} k_{1, K \mid L}^{n+1} \delta_{K, L}^{n+1}(P)$ and $-\tau_{K \mid L} k_{2, K \mid L}^{n+1} \delta_{K, L}^{n+1}(Q)$ are nondecreasing with respect to $U_{L}^{n+1}$ so that, inequalities (4.34) and (4.35) remain valid replacing $U_{L}^{n+1}$ by $U_{K}^{n+1}$. 
Thus we obtain:

$$
\begin{gathered}
k_{1}\left(U_{K}^{n+1}\right) \sum_{L \in \mathcal{N}_{K}} \tau_{K \mid L} \delta_{K, L}^{n+1}(P)+m_{K}\left(f_{1}\left(c_{K}^{n+1}\right) \bar{s}_{K}^{n+1}-f_{1}\left(U_{K}^{n+1}\right) \underline{s}_{K}^{n+1}\right)>0 \\
-k_{2}\left(U_{K}^{n+1}\right) \sum_{L \in \mathcal{N}_{K}} \tau_{K \mid L} \delta_{K, L}^{n+1}(Q)-m_{K}\left(f_{2}\left(c_{K}^{n+1}\right) \bar{s}_{K}^{n+1}-f_{2}\left(U_{K}^{n+1}\right) \underline{s}_{K}^{n+1}\right)>0 .
\end{gathered}
$$

And since $p_{c}$ is nonincreasing, we have $\delta_{K, L}^{n+1}(Q) \geq \delta_{K, L}^{n+1}(P)$, and therefore we also have

$$
-k_{2}\left(U_{K}^{n+1}\right) \sum_{L \in \mathcal{N}_{K}} \tau_{K \mid L} \delta_{K, L}^{n+1}(P)-m_{K}\left(f_{2}\left(c_{K}^{n+1}\right) \bar{s}_{K}^{n+1}-f_{2}\left(U_{K}^{n+1}\right) \underline{s}_{K}^{n+1}\right)>0 .
$$

Now let us multiply $(4.36)$ by $k_{2}\left(U_{K}^{n+1}\right),(4.38)$ by $k_{1}\left(U_{K}^{n+1}\right)$ (one of these two nonnegative values is necessarily strictly positive) and sum the two resulting inequalities. This yields:

$$
\left(k_{2}\left(U_{K}^{n+1}\right) f_{1}\left(c_{K}^{n+1}\right)-k_{1}\left(U_{K}^{n+1}\right) f_{2}\left(c_{K}^{n+1}\right)\right) m_{K} \bar{s}_{K}^{n+1}>0
$$

Now, since $k_{1}$ is nonincreasing and $k_{2}$ is nondecreasing, the left hand side in inequality (4.39) is nonincreasing with respect to $U_{K}^{n+1}$ and is equal to zero if $U_{K}^{n+1}=c_{K}^{n+1}$. This is in contradiction with the hypothesis $U_{K}^{n+1}>u_{\max }$ since $c_{K}^{n+1} \leq u_{\max }$.

\subsection{Estimates on the pressure}

The following lemma is a preliminary step to the proof of the estimates given in Proposition 4.2.

Lemma 4.1 (Preliminary step). Under assumptions and notations (2.12)-(2.21), let $D$ be a finite volume discretization of $\Omega \times(0, T)$ in the sense of Definition 3.3 and let $\left(U_{D}, P_{D}\right)$ be a solution of (3.22)-(3.26) Then the following inequalities hold:

$$
k_{1, K \mid L}^{n+1}+k_{2, K \mid L}^{n+1} \geq \alpha, \forall(K, L) \in \mathcal{E}, \forall n \in \llbracket 0, N \rrbracket,
$$

and

$$
\alpha\left(\delta_{K, L}^{n+1}(P)+\delta_{K, L}^{n+1}\left(p_{g}(U)\right)\right)^{2} \leq k_{1, K \mid L}^{n+1}\left(\delta_{K, L}^{n+1}(P)\right)^{2}+k_{2, K \mid L}^{n+1}\left(\delta_{K, L}^{n+1}(Q)\right)^{2}, \forall(K, L) \in \mathcal{E}, \forall n \in \llbracket 0, N \rrbracket
$$

(recall that $p_{g}$ is defined in (2.20)).

Proof. For the sake of clarity, we first sketch the proof of the continuous equivalent of (4.40) and (4.41). We then give in Step 2 the proof in the discrete setting, which is adapted from the continuous one.

Step 1. Proof of the continuous equivalent of (4.40) and (4.41)

The continuous equivalent of (4.40) is $k_{1}(u)+k_{2}(u) \geq \alpha$ which is the assumption (2.18) on the data.

Now the continuous equivalent of inequality (4.41) writes:

$$
\alpha\left(\nabla\left(p+p_{g}(u)\right)\right)^{2} \leq k_{1}(u)(\nabla p)^{2}+k_{2}(u)(\nabla q)^{2} .
$$

By definition, $f_{1}(u)+f_{2}(u)=1$. Hence, thanks to the Cauchy-Schwarz inequality,

$$
\begin{aligned}
\left(\nabla\left(p+p_{g}(u)\right)\right)^{2} & =\left(f_{1}(u) \nabla p+f_{2}(u) \nabla q\right)^{2} \\
& \leq f_{1}(u)(\nabla p)^{2}+f_{2}(u)(\nabla q)^{2}=\frac{k_{1}(u)(\nabla p)^{2}+k_{2}(u)(\nabla q)^{2}}{k_{1}(u)+k_{2}(u)}
\end{aligned}
$$

and (4.42) follows from the fact that $k_{1}(u)+k_{2}(u) \geq \alpha$. 
Step 2. Proof in the discrete setting

In order to prove (4.40), we separately consider the exclusive cases $(K, L) \in \mathcal{E}_{1}^{n+1} \cap \mathcal{E}_{2}^{n+1},(K, L) \notin \mathcal{E}_{1}^{n+1} \cup \mathcal{E}_{2}^{n+1}$, $(K, L) \in \mathcal{E}_{1}^{n+1}$ and $(K, L) \notin \mathcal{E}_{2}^{n+1}$, and the last case $(K, L) \notin \mathcal{E}_{1}^{n+1}$ and $(K, L) \in \mathcal{E}_{2}^{n+1}$.

If $(K, L) \in \mathcal{E}_{1}^{n+1} \cap \mathcal{E}_{2}^{n+1}$ or $(K, L) \notin \mathcal{E}_{1}^{n+1} \cup \mathcal{E}_{2}^{n+1}$, then $U_{1, K \mid L}^{n+1}=U_{2, K \mid L}^{n+1}$ holds so (4.40) is an immediate consequence of (2.18).

If $(K, L) \in \mathcal{E}_{1}^{n+1}$ and $(K, L) \notin \mathcal{E}_{2}^{n+1}$, then we have $U_{1, K \mid L}^{n+1}=U_{K}^{n+1}, U_{2, K \mid L}^{n+1}=U_{L}^{n+1}$ and $\delta_{K, L}^{n+1}\left(p_{c}(U)\right)=$ $\delta_{K, L}^{n+1}(Q)-\delta_{K, L}^{n+1}(P) \geq 0$, which yields $U_{K}^{n+1} \geq U_{L}^{n+1}$. Therefore

$$
k_{1, K \mid L}^{n+1}+k_{2, K \mid L}^{n+1} \geq k_{1}\left(U_{K}^{n+1}\right)+k_{2}\left(U_{K}^{n+1}\right) \geq \alpha .
$$

The case $(K, L) \notin \mathcal{E}_{1}^{n+1}$ and $(K, L) \in \mathcal{E}_{2}^{n+1}$ is similar. We may notice that if the upwind choice is different for the two equations, then:

$$
k_{1, K \mid L}^{n+1}=\max _{\left[U_{K}^{n+1}, U_{L}^{n+1}\right]} k_{1} \quad \text { and } \quad k_{2, K \mid L}^{n+1}=\max _{\left[U_{K}^{n+1}, U_{L}^{n+1}\right]} k_{2}
$$

Let us now turn to the proof of (4.41). Let us first study the case $(K, L) \in \mathcal{E}_{1}^{n+1}$ and $(K, L) \notin \mathcal{E}_{2}^{n+1}$. By definition of $p_{g}$ there exists some $a_{0} \in\left[U_{L}^{n+1}, U_{K}^{n+1}\right]$ such that $\delta_{K, L}^{n+1}\left(p_{g}(U)\right)=f_{2}\left(a_{0}\right) \delta_{K, L}^{n+1}\left(p_{c}(U)\right)$; hence, since $f_{1}+f_{2}=1$ and $f_{1} \leq 1, f_{2} \leq 1$, we get

$$
\begin{aligned}
\left(\delta_{K, L}^{n+1}(P)+\delta_{K, L}^{n+1}\left(p_{g}(U)\right)\right)^{2} & =\left(f_{1}\left(a_{0}\right) \delta_{K, L}^{n+1}(P)+f_{2}\left(a_{0}\right)\left(\delta_{K, L}^{n+1}(P)+\delta_{K, L}^{n+1}\left(p_{c}(U)\right)\right)\right)^{2} \\
& \leq f_{1}\left(a_{0}\right)\left(\delta_{K, L}^{n+1}(P)\right)^{2}+f_{2}\left(a_{0}\right)\left(\delta_{K, L}^{n+1}(P)+\delta_{K, L}^{n+1}\left(p_{c}(U)\right)\right)^{2}
\end{aligned}
$$

Now, from (4.43), we have $k_{1}\left(a_{0}\right) \leq k_{1, K \mid L}^{n+1}$ and $k_{2}\left(a_{0}\right) \leq k_{2, K \mid L}^{n+1}$ so that we may write:

$$
\left(\delta_{K, L}^{n+1}(P)+\delta_{K, L}^{n+1}\left(p_{g}(U)\right)\right)^{2} \leq \frac{k_{1, K \mid L}^{n+1}}{k_{1}\left(a_{0}\right)+k_{2}\left(a_{0}\right)}\left(\delta_{K, L}^{n+1}(P)\right)^{2}+\frac{k_{2, K \mid L}^{n+1}}{k_{1}\left(a_{0}\right)+k_{2}\left(a_{0}\right)}\left(\delta_{K, L}^{n+1}(P)+\delta_{K, L}^{n+1}\left(p_{c}(U)\right)\right)^{2}
$$

which gives a fortiori (4.41). The case $(K, L) \notin \mathcal{E}_{1}^{n+1}$ and $(K, L) \in \mathcal{E}_{2}^{n+1}$ is similar.

Let us now deal with the other case. If $(K, L) \in \mathcal{E}_{1}^{n+1}$ and $(K, L) \in \mathcal{E}_{2}^{n+1}$ then $k_{1, K \mid L}^{n+1}=k_{1}\left(U_{K}^{n+1}\right)$ and $k_{2, K \mid L}^{n+1}=k_{2}\left(U_{K}^{n+1}\right)$. We then remark that, since the function $f_{2}$ is nondecreasing and $p_{c}$ is nonincreasing, the following inequality holds:

$$
\begin{aligned}
k_{2}\left(U_{K}^{n+1}\right) \delta_{K, L}^{n+1}\left(p_{c}(U)\right)-\left(k_{1}\left(U_{K}^{n+1}\right)+k_{2}\left(U_{K}^{n+1}\right)\right) \delta_{K, L}^{n+1}\left(p_{g}(U)\right) & = \\
& \left(k_{1}\left(U_{K}^{n+1}\right)+k_{2}\left(U_{K}^{n+1}\right)\right) \int_{U_{K}^{n+1}}^{U_{L}^{n+1}}\left(f_{2}\left(U_{K}^{n+1}\right)-f_{2}(a)\right) p_{c}^{\prime}(a) \mathrm{d} a \leq 0 .
\end{aligned}
$$

One then gets:

$$
\begin{array}{r}
{\left[\delta_{K, L}^{n+1}(P)+\delta_{K, L}^{n+1}\left(p_{c}(U)\right)\right]\left[k_{2}\left(U_{K}^{n+1}\right) \delta_{K, L}^{n+1}\left(p_{c}(U)\right)-\left(k_{1}\left(U_{K}^{n+1}\right)+k_{2}\left(U_{K}^{n+1}\right)\right) \delta_{K, L}^{n+1}\left(p_{g}(U)\right)\right] \geq 0} \\
\delta_{K, L}^{n+1}(P)\left[k_{2}\left(U_{K}^{n+1}\right) \delta_{K, L}^{n+1}\left(p_{c}(U)\right)-\left(k_{1}\left(U_{K}^{n+1}\right)+k_{2}\left(U_{K}^{n+1}\right)\right) \delta_{K, L}^{n+1}\left(p_{g}(U)\right)\right] \geq 0 .
\end{array}
$$

Adding these two inequalities leads to:

$$
\begin{array}{r}
2 k_{2}\left(U_{K}^{n+1}\right) \delta_{K, L}^{n+1}(P) \delta_{K, L}^{n+1}\left(p_{c}(U)\right)+k_{2}\left(U_{K}^{n+1}\right)\left(\delta_{K, L}^{n+1}\left(p_{c}(U)\right)\right)^{2} \geq \\
\left(k_{1}\left(U_{K}^{n+1}\right)+k_{2}\left(U_{K}^{n+1}\right)\right)\left[2 \delta_{K, L}^{n+1}(P) \delta_{K, L}^{n+1}\left(p_{g}(U)\right)+\delta_{K, L}^{n+1}\left(p_{c}(U)\right) \delta_{K, L}^{n+1}\left(p_{g}(U)\right)\right] \geq \\
\left(k_{1}\left(U_{K}^{n+1}\right)+k_{2}\left(U_{K}^{n+1}\right)\right)\left[2 \delta_{K, L}^{n+1}(P) \delta_{K, L}^{n+1}\left(p_{g}(U)\right)+\left(\delta_{K, L}^{n+1}\left(p_{g}(U)\right)\right)^{2}\right] .
\end{array}
$$


The previous inequality gives

$$
\begin{aligned}
k_{1}\left(U_{K}^{n+1}\right)\left(\delta_{K, L}^{n+1}(P)\right)^{2}+k_{2}\left(U_{K}^{n+1}\right)\left(\delta_{K, L}^{n+1}(P)+\delta_{K, L}^{n+1}\left(p_{c}(U)\right)\right)^{2} \geq & \\
& \left(k_{1}\left(U_{K}^{n+1}\right)+k_{2}\left(U_{K}^{n+1}\right)\right)\left(\delta_{K, L}^{n+1}(P)+\delta_{K, L}^{n+1}\left(p_{g}(U)\right)\right)^{2},
\end{aligned}
$$

which is (4.41) in that case. The case $(K, L) \notin \mathcal{E}_{1}^{n+1}$ and $(K, L) \notin \mathcal{E}_{2}^{n+1}$ is similar.

Proposition 4.2 (Pressure estimates). Under assumptions and notations (2.12)-(2.21), let $D$ be a finite volume discretization of $\Omega \times(0, T)$ in the sense of Definition 3.3 and let $\left(U_{D}, P_{D}\right)$ be a solution of (3.22)-(3.26).

Then there exists $C_{1}>0$, which only depends on $k_{1}, k_{2}, p_{c}, \Omega, T, u_{0}, \bar{s}, \underline{s}$, and not on $D$, such that the following discrete $L^{2}\left(0, T ; H^{1}(\Omega)\right)$ estimates hold:

$$
\begin{aligned}
& \frac{1}{2} \sum_{n=0}^{N} \delta t^{n} \sum_{K \in \mathcal{T}} \sum_{L \in \mathcal{N}_{K}} \tau_{K \mid L} k_{1, K \mid L}^{n+1}\left(\delta_{K, L}^{n+1}(P)\right)^{2} \leq C_{1}, \\
& \frac{1}{2} \sum_{n=0}^{N} \delta t^{n} \sum_{K \in \mathcal{T}} \sum_{L \in \mathcal{N}_{K}} \tau_{K \mid L} k_{2, K \mid L}^{n+1}\left(\delta_{K, L}^{n+1}(Q)\right)^{2} \leq C_{1},
\end{aligned}
$$

and

$$
\frac{1}{2} \sum_{n=0}^{N} \delta t^{n} \sum_{K \in \mathcal{T}} \sum_{L \in \mathcal{N}_{K}} \tau_{K \mid L}\left(\delta_{K, L}^{n+1}(P)+\delta_{K, L}^{n+1}\left(p_{g}(U)\right)\right)^{2} \leq C_{1}
$$

Proof. Before proving this estimate, we shall give in Step 1 a formal proof in the continuous case to underline the main ideas.

Step 1. Proof in the continuous case

Suppose that $u$ and $p$ are regular functions that satisfy the coupled system of equations (2.5)-(2.11) and let us multiply (2.5) by $p$ and (2.6) by $q$. Then adding one equation to the other and integrating over $\Omega \times(0, T)$ yields:

$$
\begin{aligned}
\int_{0}^{T} \int_{\Omega}\left[u_{t}(x, t)\left(-p_{c}(u(x, t))\right)+k_{1}(u(x, t))(\nabla p(x, t))^{2}+k_{2}(u(x, t))(\nabla q(x, t))^{2}\right] \mathrm{d} x \mathrm{~d} t= \\
\int_{0}^{T} \int_{\Omega}\left[\left(f_{1}(c(x, t)) \bar{s}(x, t)-f_{1}(u(x, t)) \underline{s}(x, t)\right) p(x, t)+\left(f_{2}(c(x, t)) \bar{s}-f_{2}(u(x, t)) \underline{s}(x, t)\right) q(x, t)\right] \mathrm{d} x \mathrm{~d} t .
\end{aligned}
$$

Let $g_{c}$ be a primitive of $-p_{c}$. Then $\int_{0}^{T} \int_{\Omega} u_{t}(x, t)\left(-p_{c}(u(x, t))\right) \mathrm{d} x \mathrm{~d} t=\int_{\Omega}\left[g_{c}(u(x, T))-g_{c}\left(u_{0}(x, t)\right)\right] \mathrm{d} x$ which is bounded, thanks to the maximum principle. Now, thanks to Lemma 4.1, the remainder of the left hand-side of (4.46) is greater than $\alpha \int_{0}^{T} \int_{\Omega}\left(\nabla\left(p(x, t)+p_{g}(u(x, t))\right)\right)^{2} \mathrm{~d} x \mathrm{~d} t$. Hence, we may obtain a bound for $\nabla\left(p+p_{g}(u)\right)$ in $L^{2}(\Omega \times(0, T))$ provided that we control the right hand-side of (4.46). Let us then remark that $q-q_{g}(u)=$ $p+p_{g}(u)$. Hence we may write:

$$
\begin{aligned}
\left(f_{1}(c) \bar{s}-f_{1}(u) \underline{s}\right) p+\left(f_{2}(c) \bar{s}-f_{2}(u) \underline{s}\right) q= & \left(f_{1}(c) \bar{s}-f_{1}(u) \underline{s}\right)\left(p+p_{g}(u)\right)+\left(f_{2}(c) \bar{s}-f_{2}(u) \underline{s}\right)\left(q-q_{g}(u)\right) \\
& -\left(f_{1}(c) \bar{s}-f_{1}(u) \underline{s}\right) p_{g}(u)+\left(f_{2}(c) \bar{s}-f_{2}(u) \underline{s}\right) q_{g}(u) \\
= & (\bar{s}-\underline{s})\left(p+p_{g}(u)\right)+\left(f_{2}(c) \bar{s}-f_{2}(u) \underline{s}\right) q_{g}(u) \\
& -\left(f_{1}(c) \bar{s}-f_{1}(u) \underline{s}\right) p_{g}(u) .
\end{aligned}
$$


Hence, by the Poincaré-Wirtinger inequality, using the fact that $p_{g}$ and $q_{g}$ are continuous functions of $u$, and thanks the maximum principle (Prop. 4.1) and to assumption (2.17), we obtain:

$$
\left|\int_{0}^{T} \int_{\Omega}\left(f_{1}(c) \bar{s}-f_{1}(u) \underline{s}\right) p-\left(f_{2}(c) \bar{s}-f_{2}(u) \underline{s}\right) q\right| \leq C_{1}\left\|\nabla\left(p+p_{g}(u)\right)\right\|_{L^{2}(Q)}+C_{2} .
$$

Then we get a bound on $\nabla\left(p+p_{g}\right)^{2}$ in $L^{1}(\Omega \times(0, T))$ i.e. a $L^{2}\left(0, T, H^{1}(\Omega)\right)$ bound on $p+p_{g}$. Analogous bounds on $k_{1}(u) \nabla p^{2}$ and $k_{2}(u) \nabla q^{2}$ may then be obtained from equation (4.46). This completes the proof in the continuous case.

Step 2. Proof of (4.43)-(4.45) (discrete case)

In the following proof, we denote by $C_{i}$ various real values which only depend on $k_{1}, k_{2}, p_{c}, \Omega, T, u_{0}, \bar{s}, \underline{s}$, and not on $D$. Let us multiply (3.23) by $\delta t^{n} P_{K}^{n+1}$ and (3.24) by $\delta t^{n} Q_{K}^{n+1}$ and sum the two equations thus obtained. Next we sum the result over $K \in \mathcal{T}$ and $n \in \llbracket 0, N \rrbracket$. Remarking that $\sum_{K \in \mathcal{T}} \sum_{L \in \mathcal{N}_{K}}\left(P_{K}^{n+1^{2}}-P_{L}^{n+1^{2}}\right)=0$, we obtain:

$$
\begin{aligned}
-\sum_{n=0}^{N} \sum_{K \in \mathcal{T}} m_{K}\left(U_{K}^{n+1}-U_{K}^{n}\right) p_{c}\left(U_{K}^{n+1}\right)+\frac{1}{2} \sum_{n=0}^{N} \delta t^{n} \sum_{K \in \mathcal{T}} \sum_{L \in \mathcal{N}_{K}} \tau_{K \mid L} k_{1, K \mid L}^{n+1}\left|\delta_{K, L}^{n+1}(P)\right|^{2} \\
+\frac{1}{2} \sum_{n=0}^{N} \delta t^{n} \sum_{K \in \mathcal{T}} \sum_{L \in \mathcal{N}_{K}} \tau_{K \mid L} k_{2, K \mid L}^{n+1}\left|\delta_{K, L}^{n+1}(Q)\right|^{2} \leq C_{2}+\sum_{n=0}^{N} \delta t^{n} \sum_{K \in \mathcal{T}} m_{K}\left(\bar{s}_{K}^{n+1}-\underline{s}_{K}^{n+1}\right) P_{K}^{n+1}
\end{aligned}
$$

Let $g_{c} \in \mathcal{C}^{1}\left([0,1], \mathbb{R}_{+}\right)$be the function defined by $g_{c}(b)=\int_{b}^{1} p_{c}(a) \mathrm{d} a, \forall b \in[0,1]$. Since $p_{c}$ is a decreasing function, the function $g_{c}$ is convex. We thus get:

$$
-\left(U_{K}^{n+1}-U_{K}^{n}\right) p_{c}\left(U_{K}^{n+1}\right) \geq g_{c}\left(U_{K}^{n+1}\right)-g_{c}\left(U_{K}^{n}\right), \forall K \in \mathcal{T}, \forall n \in \mathbb{N} .
$$

Let us now consider the right hand-side. Let us remark that:

$$
\begin{aligned}
\sum_{n=0}^{N} \delta t^{n} \sum_{K \in \mathcal{T}} m_{K}\left(\bar{s}_{K}^{n+1}-\underline{s}_{K}^{n+1}\right) P_{K}^{n+1}= & \sum_{n=0}^{N} \delta t^{n} \sum_{K \in \mathcal{T}} m_{K}\left(\bar{s}_{K}^{n+1}-\underline{s}_{K}^{n+1}\right)\left(P_{K}^{n+1}+p_{g}\left(U_{K}^{n+1}\right)\right) \\
& -\sum_{n=0}^{N} \delta t^{n} \sum_{K \in \mathcal{T}} m_{K}\left(\bar{s}_{K}^{n+1}-\underline{s}_{K}^{n+1}\right) p_{g}\left(U_{K}^{n+1}\right) .
\end{aligned}
$$

Hence, by Proposition 4.1, assumption (2.17), and by the discrete Poincaré-Wirtinger inequality, we get that:

$$
\sum_{n=0}^{N} \delta t^{n} \sum_{K \in \mathcal{T}} m_{K}\left(\bar{s}_{K}^{n+1}-\underline{s}_{K}^{n+1}\right) P_{K}^{n+1} \leq C_{2}\left(\frac{1}{2} \sum_{n=0}^{N} \delta t^{n} \sum_{K \in \mathcal{T}} \sum_{L \in \mathcal{N}_{K}} \tau_{K \mid L}\left(\delta_{K, L}^{n+1}(P)+\delta_{K, L}^{n+1}\left(p_{g}(U)\right)\right)^{2}\right)^{1 / 2}+C_{3} .
$$

Thanks to Young's inequality, this implies the existence of $C_{4}$ such that:

$$
\sum_{n=0}^{N} \delta t^{n} \sum_{K \in \mathcal{T}} m_{K}\left(\bar{s}_{K}^{n+1}-\underline{s}_{K}^{n+1}\right) P_{K}^{n+1} \leq \frac{\alpha}{4} \sum_{n=0}^{N} \delta t^{n} \sum_{K \in \mathcal{T}} \sum_{L \in \mathcal{N}_{K}} \tau_{K \mid L}\left(\delta_{K, L}^{n+1}(P)+\delta_{K, L}^{n+1}\left(p_{g}(U)\right)\right)^{2}+C_{4} .
$$

Inequalities (4.41), (4.47), (4.48) and (4.49) give (4.45). 


\subsection{Existence of a discrete solution}

We prove here the existence of a solution to the scheme, which is a consequence of the invariance by homotopy of the Brower topological degree. This technique was first used for the existence of a solution to a nonlinear discretization scheme in [21]. The idea of the proof is the following: if we can modify continuously the scheme to obtain a linear system and if the modification simultaneously preserves the estimates which were obtained in Propositions 4.1 and 4.2, then the scheme has at least one solution (since in the linear case, these estimates also prove that the linear system has a unique solution).

Proposition 4.3. Under Hypothesis (2.14)-(2.19), there exists at least one solution $\left(U_{D}, P_{D}\right)$ to the scheme (3.22)-(3.26)

Proof. We define the vector space of discrete solutions $E_{D}$ by

$$
E_{D}=\mathbb{R}^{\mathcal{T} \times \llbracket 0, N+1 \rrbracket} \times \mathbb{R}^{\mathcal{T} \times \llbracket 1, N+1 \rrbracket} .
$$

Let $K_{0}$ be a given control volume of the mesh. We define a continuous application $\mathcal{F}:[0,1] \times E_{D} \rightarrow E_{D}$ by $\mathcal{F}\left(t,\left(U_{D}, P_{D}\right)\right)=\left(A_{D}, B_{D}\right)$, where

$$
\left\{\begin{aligned}
A_{K}^{0}= & U_{K}^{0}-\frac{1}{m_{K}} \int_{K} u_{0}(x) \mathrm{d} x, \quad \forall K \in \mathcal{T}, \\
A_{K}^{n+1}= & \frac{U_{K}^{n+1}-U_{K}^{n}}{\delta t^{n}} m_{K}-\sum_{L \in \mathcal{N}_{K}} \tau_{K \mid L} k_{1 K \mid L}^{t} \delta_{K, L}^{n+1} \delta^{n+1}(P) \\
& -m_{K}\left(f^{t}\left(c_{K}^{n+1}\right) t \bar{s}_{K}^{n+1}+f^{t}\left(U_{K}^{n+1}\right) t \underline{s}_{K}^{n+1}\right), \quad \forall(K, n) \in \mathcal{T} \times \llbracket 0, N \rrbracket, \\
B_{K}^{n+1}= & \frac{U_{K}^{n}-U_{K}^{n+1}}{\delta t^{n}} m_{K}-\sum_{L \in \mathcal{N}_{K}} \tau_{K \mid L} k_{2 K \mid L}^{t}{ }^{n+1}\left(\delta_{K, L}^{n+1}(P)+\delta_{K, L}^{n+1}\left(p_{c}{ }^{t}(U)\right)\right) \\
& \left.-m_{K}\left(h^{t}\left(c_{K}^{n+1}\right) t \bar{s}_{K}^{n+1}+h^{t}\left(U_{K}^{n+1}\right)\right) t \underline{s}_{K}^{n+1}\right), \quad \forall K \in \mathcal{T} \backslash K_{0}, \quad \forall n \in \llbracket 0, N \rrbracket, \\
B_{K_{0}}^{n+1}= & \sum_{K \in \mathcal{T}} m_{K} P_{K}^{n+1}, \quad \forall n \in \llbracket 0, N \rrbracket .
\end{aligned}\right.
$$

In (4.50), $u_{0}^{t}, k_{1}^{t}, k_{2}^{t}, f^{t}$ and $p_{c}{ }^{t}$ are continuous modifications of $u_{0}, k_{1}, k_{2}, f_{1}$ and $p_{c}$ which preserve the properties used to obtain the maximum principle and the pressure estimates. More precisely, we take $x_{0} \in[0,1]$, we denote by $H^{t}$ the function defined by $H^{t}(x)=t x+(1-t)\left(x_{0}\right)$ and we choose $k_{1}^{t}=k_{1} \circ H^{t}, k_{2}^{t}=k_{2} \circ H^{t}, f^{t}=f \circ H^{t}$ and $p_{c}{ }^{t}=p_{c} \circ H^{t}$. Then, the definition of $k_{1}^{t}(u)_{K \mid L}^{n+1}$ and $k_{2}^{t}(u)_{K \mid L}^{n+1}$ is the analogue of definition of $k_{1, K \mid L}^{n+1}$ and $k_{2, K \mid L}^{n+1}$ by (3.27) with $k_{1}^{t}, k_{2}^{t}$ and $Q^{t}$ instead of $k_{1}, k_{2}$ and $Q$, with $\delta_{K, L}^{n+1}\left(Q^{t}\right)=\delta_{K, L}^{n+1}(P)+\delta_{K, L}^{n+1}\left(p_{c}{ }^{t}(U)\right)$.

Let us now complete the proof. First of all, $\mathcal{F}(0, \cdot)$ is clearly an affine function. Moreover $\mathcal{F}\left(t,\left(U_{D}, P_{D}\right)\right)=0$ if and only if $\left(U_{D}, P_{D}\right)$ is a solution to the scheme with functions $u_{0}^{t}, k_{1}^{t}, k_{2}^{t}, f^{t}, p_{c}{ }^{t}, t \bar{s}$, and $t \underline{s}$. Indeed, thanks to assumption (2.17), we have that: $\sum_{K \in \mathcal{T}}\left(\bar{s}_{K}^{n+1}-\underline{s}_{K}^{n+1}\right)=0$. Therefore, the equation of (3.22)(3.26) corresponding to the finite volume scheme for the conservation of component 2 in the control volume $K=K_{0}$ may be obtained by summing all the equations of (4.50) corresponding to the other control volumes. Hence, using the a priori estimates (4.33) and (4.43) and the discrete Poincaré-Wirtinger inequality, we get a bound on $U_{D}$ and $P_{D}$ independent of $t$. The function $\mathcal{F}$ is continuous. Indeed, the terms corresponding to the phase by phase upwinding can be rewritten with the help of the continuous functions $x \mapsto x^{+}=\max (x, 0)$ and $x \mapsto x^{-}=\max (-x, 0)$ in the following way: $k_{i K \mid L}^{n+1} \delta_{K, L}^{n+1}(P)=k_{i}^{t}\left(U_{K}^{n+1}\right)\left(\delta_{K, L}^{n+1}(P)\right)^{+}-k_{i}^{t}\left(U_{L}^{n+1}\right)\left(\delta_{K, L}^{n+1}(P)\right)^{-}$for $i=1,2$. If $X$ is a ball with a sufficiently large radius in $E_{D}$, the equation $\mathcal{F}\left(t,\left(U_{D}, P_{D}\right)\right)=0$ has no solution on the boundary of $X$, so that

$$
\operatorname{degree}(\mathcal{F}(1, \cdot), X)=\operatorname{degree}(\mathcal{F}(0, \cdot), X)=\operatorname{det}(\mathcal{F}(0, \cdot)) \neq 0
$$


where "degree" denotes the Brower topological degree (see e.g. [15]). Hence by the property of invariance by homotopy of the Brower degree, we obtain the existence of at least one solution to the scheme.

\subsection{Estimates on $\mathbf{g}(\mathbf{u})$}

The following estimate is first used below to prove a compactness property on $U_{D}$, and then used for the convergence result. The proof in the continuous case is not very difficult, but it strongly uses the symmetry of the system. The discrete proof is somewhat more complicated because of the phase by phase upstream weighting.

Proposition 4.4. Under assumptions and notations (2.12)-(2.21), let $D$ be a finite volume discretization of $\Omega \times(0, T)$ in the sense of Definition 3.3 and let $\left(U_{D}, P_{D}\right)$ be a solution of the finite volume scheme (3.22)-(3.26) Then there exists $C_{5}$, which only depends on $k_{1}, k_{2}, p_{c}, \Omega, T, u_{0}, \bar{s}, \underline{s}$, and not on $D$, such that the following discrete $L^{2}\left(0, T ; H^{1}(\Omega)\right)$ estimate holds:

$$
\sum_{n=0}^{N} \delta t^{n} \sum_{K \in \mathcal{T}} \sum_{L \in \mathcal{N}_{K}} \tau_{K \mid L} \delta_{K, L}^{n+1}(g(U)) \delta_{K, L}^{n+1}\left(f_{1}(U)\right) \leq C_{5}
$$

Proof. Step 1. Proof in the continuous case

Let us first sketch the proof in the continuous case, assuming that $(u, p)$ is a regular solution. The continuous estimate to (4.51) writes:

$$
\int_{0}^{T} \int_{\Omega} \nabla g(u(x, t)) \nabla f_{1}(u(x, t)) \mathrm{d} x \mathrm{~d} t \leq C .
$$

To preserve the symmetry of the system, we multiply the first equation by $f_{1}(u)$ and the second equation by $f_{2}(u)$. Summing the two equations we obtain:

$$
\begin{array}{r}
\int_{0}^{T} \int_{\Omega}\left[u_{t}(x, t)\left(f_{1}(u(x, t))-f_{2}(u(x, t))\right)-\operatorname{div}\left(k_{1}(u(x, t)) \nabla p(x, t)\right) f_{1}(u(x, t))\right. \\
\left.-\operatorname{div}\left(k_{2}(u(x, t)) \nabla q(x, t)\right) f_{2}(u(x, t))\right] \mathrm{d} x \mathrm{~d} t=\int_{0}^{T} \int_{\Omega} f_{1}(u(x, t))\left(f_{1}(c(x, t)) \bar{s}(x, t)-f_{1}(u(x, t)) \underline{s}(x, t)\right) \\
+\int_{0}^{T} \int_{\Omega} f_{2}(u(x, t))\left(f_{2}(c(x, t)) \bar{s}(x, t)-f_{2}(u(x, t)) \underline{s}(x, t)\right) .
\end{array}
$$

Let us introduce the total velocity flow $F$ which writes: $F=k_{1}(u) \nabla p+k_{2}(u) \nabla q$. Remarking that $k_{2}(u) p_{c}{ }^{\prime}(u) \nabla u=$ $\left(k_{1}(u)+k_{2}(u)\right) \nabla p_{g}(u)$, one has: $F=\left(k_{1}(u)+k_{2}(u)\right) \nabla\left(p+p_{g}(u)\right)$ and $F=\left(k_{1}(u)+k_{2}(u)\right) \nabla\left(q-q_{g}(u)\right)$, so that $k_{1}(u) \nabla p=f_{1}(u) F-k_{1}(u) \nabla p_{g}(u)$ and $k_{2}(u) \nabla q=f_{2}(u) F+k_{2}(u) \nabla q_{g}(u)$. By definition of $p_{g}, q_{g}$, and $g$ (see $(2.20,2.21))$, one also has: $k_{1}(u) \nabla p_{g}(u)=k_{2}(u) \nabla q_{g}(u)=-\nabla g(u)$. Hence:

$$
\begin{array}{r}
\int_{0}^{T} \int_{\Omega}\left[u_{t}(x, t)\left(f_{1}(u(x, t))-f_{2}(u)(x, t)\right)\right] \mathrm{d} x \mathrm{~d} t-\int_{0}^{T} \int_{\Omega}\left[\operatorname{div}\left(f_{1}(u(x, t)) F(x, t)\right) f_{1}(u(x, t))\right. \\
\left.+\operatorname{div}\left(f_{2}(u(x, t)) F(x, t)\right) f_{2}(u(x, t))\right] \mathrm{d} x \mathrm{~d} t-\int_{0}^{T} \int_{\Omega} \Delta g(u(x, t))\left(f_{1}(u(x, t))-f_{2}(u(x, t))\right) \mathrm{d} x \mathrm{~d} t= \\
\int_{0}^{T} \int_{\Omega}\left[f_{1}(u(x, t))\left(f_{1}(c(x, t)) \bar{s}(x, t)-f_{1}(u(x, t)) \underline{s}(x, t)\right)\right. \\
\left.+f_{2}(u(x, t))\left(f_{2}(c(x, t)) \bar{s}(x, t)-f_{2}(u(x, t)) \underline{s}(x, t)\right)\right] \mathrm{d} x \mathrm{~d} t .
\end{array}
$$


The right hand-side of this equation is clearly bounded. The first term in the left side is also bounded (consider for example a primitive of $f_{1}(u)-f_{2}(u)$ ). Assuming that there exists a bound to the second term, an integration by parts in the third term and the fact that $\nabla f_{1}(u)=-\nabla f_{2}(u)$ yield (4.52). Let us then deal with the second term (and the third) of the left hand side, i.e. the term concerning $F$. By summing equations (2.5) and (2.6), we obtain that $\operatorname{div}(F)=\bar{s}-\underline{s}$, so that $\operatorname{div}(F)$ is bounded in $L^{2}(\Omega \times(0, T))$. Moreover, one has: $\operatorname{div}\left(f_{i}(u) F\right) f_{i}(u)=$ $\frac{1}{2} \operatorname{div}\left(f_{i}(u)^{2} F\right)+\frac{1}{2} f_{i}(u)^{2} \operatorname{div} F$, for $i=1,2$, and since $F \cdot n=0$ on $\partial \Omega, \int_{\Omega \times(0, T)} \operatorname{div}\left(f_{i}(u)^{2} F\right)=0$ for $i=1,2$. Hence we get a bound for the second and the third term of the left hand side of (4.53). This completes the proof in the continuous case.

Step 2. The discrete counterpart: proof of (4.51)

In the following proof, we denote by $C_{i}$ various real values which only depend on $k_{1}, k_{2}, p_{c}, \Omega, T, u_{0}, \bar{s}, \underline{s}$, and not on $D$. Let us multiply (3.23) by $\delta t^{n} f_{1}\left(U_{K}^{n+1}\right)$ and $(3.24)$ by $\delta t^{n} f_{2}\left(U_{K}^{n+1}\right)$ and sum the two equations thus obtained. Next we sum the result over $K \in \mathcal{T}$ and $n \in \llbracket 0, N \rrbracket$. This yields:

$$
\begin{array}{r}
\sum_{n=0}^{N} \sum_{K \in \mathcal{T}} m_{K}\left(U_{K}^{n+1}-U_{K}^{n}\right)\left(f_{1}\left(U_{K}^{n+1}\right)-f_{2}\left(U_{K}^{n+1}\right)\right)-\sum_{n=0}^{N} \delta t^{n} \sum_{K \in \mathcal{T}} f_{1}\left(U_{K}^{n+1}\right) \sum_{L \in \mathcal{N}_{K}} \tau_{K \mid L} k_{1, K \mid L}^{n+1} \delta_{K, L}^{n+1}(P) \\
-\sum_{n=0}^{N} \delta t^{n} \sum_{K \in \mathcal{T}} f_{2}\left(U_{K}^{n+1}\right) \sum_{L \in \mathcal{N}_{K}} \tau_{K \mid L} k_{2, K \mid L}^{n+1} \delta_{K, L}^{n+1}(Q) \leq C_{7} .
\end{array}
$$

Adding (3.23) and (3.24) gives

$$
\sum_{L \in \mathcal{N}_{K}} \tau_{K \mid L} F_{K, L}^{n+1}=m_{K}\left(\bar{s}_{K}^{n+1}-\underline{s}_{K}^{n+1}\right)
$$

where $F_{K, L}^{n+1}$ is the discrete counterpart of the total flux $F$, that is:

$$
\begin{aligned}
F_{K, L}^{n+1} & =-k_{1, K \mid L}^{n+1} \delta_{K, L}^{n+1}(P)-k_{2, K \mid L}^{n+1} \delta_{K, L}^{n+1}(Q) \\
& =-\left(k_{1, K \mid L}^{n+1}+k_{2, K \mid L}^{n+1}\right) \delta_{K, L}^{n+1}(P)-k_{2, K \mid L}^{n+1} \delta_{K, L}^{n+1}\left(p_{c}(U)\right) \\
& =-\left(k_{1, K \mid L}^{n+1}+k_{2, K \mid L}^{n+1}\right) \delta_{K, L}^{n+1}(Q)+k_{1, K \mid L}^{n+1} \delta_{K, L}^{n+1}\left(p_{c}(U)\right) .
\end{aligned}
$$

The first step of the estimate follows the continuous case; the total velocity flux $F$ and the function $g(u)$ are introduced by writing $k_{1} \nabla P$ as a function of $F$ and $\nabla p_{c}(u)$. In the discrete case, the values of $U_{1, K \mid L}^{n+1}$ and $U_{2, K \mid L}^{n+1}$ may differ. Hence we shall need to decompose the numerical fluxes $k_{i, K \mid L}^{n+1} \delta_{K, L}^{n+1}(P)$ for $i=1$, 2, in the following way: $k_{i, K \mid L}^{n+1} \delta_{K, L}^{n+1}(P)=-f_{i}\left(U_{i, K \mid L}^{n+1}\right) F_{K, L}^{n+1}+\Phi_{i, K, L}^{n+1}+R_{i, K, L}^{n+1}$ with $\Phi_{i, K, L}^{n+1}=-f_{i}\left(U_{i, K \mid L}^{n+1}\right) k_{j, K \mid L}^{n+1} \delta_{K, L}^{n+1}\left(p_{c}(U)\right)$, and $R_{i, K, L}^{n+1}=f_{i}\left(U_{i, K \mid L}^{n+1}\right)\left[k_{j}\left(U_{i, K \mid L}^{n+1}\right)-k_{j}\left(U_{j, K \mid L}^{n+1}\right)\right] \delta_{K, L}^{n+1}(P)$, with $j=1,2, j \neq i$. In order to deal with the time derivative terms, we once more use the inequality $(b-a) G^{\prime}(b) \geq G(b)-G(a)$ for any functions $G$ such that $G^{\prime}=f_{1}-f_{2}$ (which is therefore convex since $f_{1}-f_{2}$ is nondecreasing), and get:

$$
-\sum_{n=0}^{N} \delta t^{n} \sum_{K \in \mathcal{T}} m_{K}\left(U_{K}^{n+1}-U_{K}^{n}\right)\left(f_{1}\left(U_{K}^{n+1}\right)-f_{2}\left(U_{K}^{n+1}\right)\right) \leq \sum_{K \in \mathcal{T}} m_{K}\left(G\left(U_{K}^{N+1}\right)-G\left(U_{K}^{0}\right)\right) \leq C_{8} .
$$


Gathering by edges, and remarking that $\delta_{K, L}^{n+1}\left(f_{1}(U)\right)+\delta_{K, L}^{n+1}\left(f_{2}(U)\right)=0$ (this is a direct consequence of $f_{1}+f_{2}=$ 1), we then obtain:

$$
\begin{aligned}
\sum_{n=0}^{N} \delta t^{n} \sum_{K \in \mathcal{T}} f_{1}\left(U_{K}^{n+1}\right) & \sum_{L \in \mathcal{N}_{K}} \tau_{K \mid L} f_{1}\left(U_{1, K \mid L}^{n+1}\right) F_{K, L}^{n+1}+\sum_{n=0}^{N} \delta t^{n} \sum_{K \in \mathcal{T}} f_{2}\left(U_{K}^{n+1}\right) \sum_{L \in \mathcal{N}_{K}} \tau_{K \mid L} f_{2}\left(U_{2, K \mid L}^{n+1}\right) F_{K, L}^{n+1} \\
& +\frac{1}{2} \sum_{n=0}^{N} \delta t^{n} \sum_{K \in \mathcal{T}} \sum_{L \in \mathcal{N}_{K}} \tau_{K \mid L}\left(\Phi_{1, K, L}^{n+1}+\Phi_{2, K, L}^{n+1}+R_{1, L, K}^{n+1}+R_{2, L, K}^{n+1}\right) \delta_{K, L}^{n+1}\left(f_{1}(U)\right) \leq C_{8} .
\end{aligned}
$$

Since $f_{1}+f_{2}=1$, multiplying (4.54) by $f_{1}\left(U_{K}^{n+1}\right)+f_{2}\left(U_{K}^{n+1}\right)$, summing over $K \in \mathcal{T}$ and substracting from (4.56) yields:

$$
\begin{array}{r}
\sum_{n=0}^{N} \delta t^{n} \sum_{K \in \mathcal{T}} f_{1}\left(U_{K}^{n+1}\right) \sum_{L \in \mathcal{N}_{K}} \tau_{K \mid L}\left(f_{1}\left(U_{1, K \mid L}^{n+1}\right)-f_{1}\left(U_{K}^{n+1}\right)\right) F_{K, L}^{n+1}+\sum_{n=0}^{N} \delta t^{n} \sum_{K \in \mathcal{T}} f_{2}\left(U_{K}^{n+1}\right) \sum_{L \in \mathcal{N}_{K}} \tau_{K \mid L}\left(f_{2}\left(U_{2, K \mid L}^{n+1}\right)\right. \\
\left.-f_{2}\left(U_{K}^{n+1}\right)\right) F_{K, L}^{n+1}+\frac{1}{2} \sum_{n=0}^{N} \delta t^{n} \sum_{K \in \mathcal{T}} \sum_{L \in \mathcal{N}_{K}} \tau_{K \mid L}\left(\Phi_{1, K, L}^{n+1}+\Phi_{2, K, L}^{n+1}+R_{1, L, K}^{n+1}+R_{2, L, K}^{n+1}\right) \delta_{K, L}^{n+1}\left(f_{1}(U)\right) \leq C_{9} .
\end{array}
$$

Using the equality $b(a-b)=-\frac{1}{2}(a-b)^{2}+\frac{1}{2}\left(a^{2}-b^{2}\right)$, we get from (4.57),

$$
\begin{aligned}
& -\frac{1}{2} \sum_{n=0}^{N} \delta t^{n} \sum_{K \in \mathcal{T}} \sum_{\substack{L \in \mathcal{N}_{K} \\
(K, L) \notin \mathcal{E}_{1}^{n+1}}} \tau_{K \mid L}\left(f_{1}\left(U_{K}^{n+1}\right)-f_{1}\left(U_{L}^{n+1}\right)\right)^{2} F_{K, L}^{n+1} \\
& \quad+\frac{1}{2} \sum_{n=0}^{N} \delta t^{n} \sum_{K \in \mathcal{T}} \sum_{\substack{L \in \mathcal{N}_{K} \\
(K, L) \notin \mathcal{E}_{1}^{n+1}}} \tau_{K \mid L}\left(f^{2}\left(U_{K}^{n+1}\right)-f^{2}\left(U_{L}^{n+1}\right)\right) F_{K, L}^{n+1} \\
& \\
& \quad-\frac{1}{2} \sum_{n=0}^{N} \delta t^{n} \sum_{K \in \mathcal{T}} \sum_{\substack{L \in \mathcal{N}_{K} \\
(K, L) \notin \mathcal{E}_{2}^{n+1}}} \tau_{K \mid L}\left(f_{2}\left(U_{K}^{n+1}\right)-f_{2}\left(U_{L}^{n+1}\right)\right)^{2} F_{K, L}^{n+1} \\
& \quad+\frac{1}{2} \sum_{n=0}^{N} \delta t^{n} \sum_{K \in \mathcal{T}} \sum_{\substack{L \in \mathcal{N}_{K} \\
(K, L) \notin \mathcal{E}_{2}^{n+1}}} \tau_{K \mid L}\left(h^{2}\left(U_{K}^{n+1}\right)-h^{2}\left(U_{L}^{n+1}\right)\right) F_{K, L}^{n+1} \\
& \quad+\frac{1}{2} \sum_{n=0}^{N} \delta t^{n} \sum_{K \in \mathcal{T}} \sum_{L \in \mathcal{N}_{K}} \tau_{K \mid L}\left(\Phi_{1, K, L}^{n+1}+\Phi_{2, K, L}^{n+1}+R_{1, L, K}^{n+1}+R_{2, L, K}^{n+1}\right) \delta_{K, L}^{n+1}\left(f_{1}(U)\right) \leq C_{9} .
\end{aligned}
$$

If we denote by $B_{2}$ and $B_{4}$ the second and fourth terms in (4.58), we have:

$$
B_{2}=\frac{1}{2} \sum_{n=0}^{N} \delta t^{n} \sum_{K \in \mathcal{T}}\left(f_{1}\left(U_{K}^{n+1}\right)\right)^{2}\left(\sum_{\substack{L \in \mathcal{N}_{K} \\(K, L) \notin \mathcal{E}_{1}^{n+1}}} \tau_{K \mid L} F_{K, L}^{n+1}-\sum_{\substack{L \in \mathcal{N}_{K} \\(L, K) \notin \mathcal{E}_{1}^{n+1}}} \tau_{K \mid L} F_{L, K}^{n+1}\right)
$$


But $(L, K) \notin \mathcal{E}_{1}^{n+1} \Leftrightarrow(K, L) \in \mathcal{E}_{1}^{n+1}$ and $F_{K, L}=-F_{L, K}$, so that:

$$
B_{2}=\frac{1}{2} \sum_{n=0}^{N} \delta t^{n} \sum_{K \in \mathcal{T}}\left(f_{1}\left(U_{K}^{n+1}\right)\right)^{2} \sum_{L \in \mathcal{N}_{K}} \tau_{K \mid L} F_{K, L}^{n+1} \leq C_{6},
$$

and in the same way $B_{4} \leq C_{11}$. Therefore if we develop all the terms, we obtain:

$$
\begin{aligned}
\frac{1}{2} \sum_{n=0}^{N} \delta t^{n} & \sum_{\substack{(K, L) \in \mathcal{E}_{1}^{n+1} \\
(K, L) \in \mathcal{E}_{2}^{n+1}}} \tau_{K \mid L}\left|\delta_{K, L}^{n+1}\left(f_{1}(U)\right)\right|^{2}\left(F_{K, L}^{n+1}+F_{K, L}^{n+1}\right)+\frac{1}{2} \sum_{n=0}^{N} \delta t^{n} \sum_{\substack{(K, L) \in \mathcal{E}_{1}^{n+1} \\
(K, L) \notin \mathcal{E}_{2}^{n+1}}} \tau_{K \mid L}\left|\delta_{K, L}^{n+1}\left(f_{1}(U)\right)\right|^{2}\left(F_{K, L}^{n+1}+F_{L, K}^{n+1}\right) \\
& +\sum_{n=0}^{N} \delta t^{n} \sum_{(K, L) \in \mathcal{E}_{1}^{n+1}} \tau_{K \mid L}\left(\Phi_{1, K, L}^{n+1}+\Phi_{2, K, L}^{n+1}+R_{1, L, K}^{n+1}+R_{2, L, K}^{n+1}\right) \delta_{K, L}^{n+1}\left(f_{1}(U)\right) \leq C_{7}
\end{aligned}
$$

Since $F_{K, L}^{n+1}+F_{L, K}^{n+1}=0,(4.59)$ leads to:

$$
\sum_{n=0}^{N} \delta t^{n} \sum_{(K, L) \in \mathcal{E}_{1}^{n+1}} \tau_{K \mid L} D_{K, L}^{n+1} \leq C_{8}
$$

where

$$
\begin{aligned}
& D_{K, L}^{n+1}=\left|\delta_{K, L}^{n+1}\left(f_{1}(U)\right)\right|^{2} F_{K, L}^{n+1}+\left(\Phi_{1, K, L}^{n+1}+\Phi_{2, K, L}^{n+1}+R_{1, L, K}^{n+1}+R_{2, L, K}^{n+1}\right) \delta_{K, L}^{n+1}\left(f_{1}(U)\right), \\
& \forall(K, L) \in \mathcal{E}_{1}^{n+1},(K, L) \in \mathcal{E}_{2}^{n+1}
\end{aligned}
$$

and

$$
D_{K, L}^{n+1}=\left(\Phi_{1, K, L}^{n+1}+\Phi_{2, K, L}^{n+1}+R_{1, L, K}^{n+1}+R_{2, L, K}^{n+1}\right) \delta_{K, L}^{n+1}\left(f_{1}(U)\right), \forall(K, L) \in \mathcal{E}_{1}^{n+1},(K, L) \notin \mathcal{E}_{2}^{n+1} .
$$

Let us first study $D_{K, L}^{n+1}$ for $(K, L) \in \mathcal{E}_{1}^{n+1} \cap \mathcal{E}_{2}^{n+1}$. Since $U_{1, K \mid L}^{n+1}=U_{2, K \mid L}^{n+1}=U_{K}^{n+1}$, it is clear that $R_{1, L, K}^{n+1}=$ $R_{2, L, K}^{n+1}=0$ and we have

$$
D_{K, L}^{n+1}=\delta_{K, L}^{n+1}\left(f_{1}(U)\right)\left(\delta_{K, L}^{n+1}\left(f_{1}(U)\right) F_{K, L}^{n+1}-2 \frac{k_{1}\left(U_{K}^{n+1}\right) k_{2}\left(U_{K}^{n+1}\right)}{k_{1}\left(U_{K}^{n+1}\right)+k_{2}\left(U_{K}^{n+1}\right)} \delta_{K, L}^{n+1}\left(p_{c}(U)\right)\right) .
$$

If we assume that $U_{K}^{n+1} \leq U_{L}^{n+1}$, then $\delta_{K, L}^{n+1}\left(p_{c}(U)\right) \leq 0$ and $F_{K, L}^{n+1} \geq-k_{2}\left(U_{K}^{n+1}\right)\left(\delta_{K, L}^{n+1}\left(p_{c}(U)\right)\right)$, which leads to

$$
\begin{aligned}
D_{K, L}^{n+1} & \geq-\left[\delta_{K, L}^{n+1}\left(f_{1}(U)\right) k_{2}\left(U_{K}^{n+1}\right)+2 \frac{k_{1}\left(U_{K}^{n+1}\right) k_{2}\left(U_{K}^{n+1}\right)}{k_{1}\left(U_{K}^{n+1}\right)+k_{2}\left(U_{K}^{n+1}\right)}\right] \delta_{K, L}^{n+1}\left(f_{1}(U)\right) \delta_{K, L}^{n+1}\left(p_{c}(U)\right) \\
& \geq-\left[f_{1}\left(U_{K}^{n+1}\right)+f_{1}\left(U_{L}^{n+1}\right)\right] k_{2}\left(U_{K}^{n+1}\right) \delta_{K, L}^{n+1}\left(f_{1}(U)\right) \delta_{K, L}^{n+1}\left(p_{c}(U)\right) .
\end{aligned}
$$

And since $f_{1}\left(U_{K}^{n+1}\right) \geq 0$, we get that:

$$
\begin{aligned}
D_{K, L}^{n+1} & \geq-\frac{k_{1}\left(U_{L}^{n+1}\right) k_{2}\left(U_{K}^{n+1}\right)}{k_{1}\left(U_{L}^{n+1}\right)+k_{2}\left(U_{L}^{n+1}\right)} \delta_{K, L}^{n+1}\left(f_{1}(U)\right) \delta_{K, L}^{n+1}\left(p_{c}(U)\right) \\
& \geq \delta_{K, L}^{n+1}\left(f_{1}(U)\right) \int_{U_{K}^{n+1}}^{U_{L}^{n+1}} \frac{k_{1}(a) k_{2}(a)}{k_{1}(a)+k_{2}(a)}\left(-p_{c}{ }^{\prime}(a)\right) \mathrm{d} a=\delta_{K, L}^{n+1}\left(f_{1}(U)\right) \delta_{K, L}^{n+1}(g(U)) .
\end{aligned}
$$


The same inequality holds in the case $U_{K}^{n+1} \geq U_{L}^{n+1}$, and is obtained similarly, changing the roles of $k_{1}$ and $k_{2}$, and $f_{1}$ and $f_{2}$.

We now study $D_{K, L}^{n+1}$ for $(K, L) \in \mathcal{E}_{1}^{n+1},(K, L) \notin \mathcal{E}_{2}^{n+1}$. We have $\delta_{K, L}^{n+1}(P) \leq 0$ and $\delta_{K, L}^{n+1}(Q) \geq 0$, which yields $\delta_{K, L}^{n+1}\left(p_{c}(U)\right) \geq 0$, and therefore $U_{K}^{n+1} \geq U_{L}^{n+1}$. We have:

$$
\begin{aligned}
D_{K, L}^{n+1}= & -\delta_{K, L}^{n+1}\left(f_{1}(U)\right)\left[f_{1}\left(U_{K}^{n+1}\right) k_{2}\left(U_{L}^{n+1}\right) \delta_{K, L}^{n+1}\left(p_{c}(U)\right)+\delta_{K, L}^{n+1}\left(k_{2}(U)\right) \delta_{K, L}^{n+1}(P)\right. \\
& +f_{2}\left(U_{L}^{n+1}\right)\left(k_{1}\left(U_{L}^{n+1}\right) \delta_{K, L}^{n+1}\left(p_{c}(U)\right)+\delta_{K, L}^{n+1}\left(k_{1}(U)\right) \delta_{K, L}^{n+1}(Q)\right]
\end{aligned}
$$

Now we use the symmetry of the problem in $p$ and $q$. We can express $\delta_{K, L}^{n+1}(Q)$ as a function of $\delta_{K, L}^{n+1}(P)$ or conversely. In the first case we obtain:

$$
\begin{aligned}
D_{K, L}^{n+1}= & -\left[\left(f_{1}\left(U_{K}^{n+1}\right) k_{2}\left(U_{L}^{n+1}\right)+f_{2}\left(U_{L}^{n+1}\right)\left(k_{1}\left(U_{L}^{n+1}\right)\right)\right] \delta_{K, L}^{n+1}\left(f_{1}(U)\right) \delta_{K, L}^{n+1}\left(p_{c}(U)\right)\right. \\
& -\left[f_{1}\left(U_{K}^{n+1}\right) \delta_{K, L}^{n+1}\left(k_{2}(U)\right)+f_{2}\left(U_{L}^{n+1}\right) \delta_{K, L}^{n+1}\left(k_{1}(U)\right)\right] \delta_{K, L}^{n+1}\left(f_{1}(U)\right) \delta_{K, L}^{n+1}(P) .
\end{aligned}
$$

In the second case, we obtain:

$$
\begin{aligned}
D_{K, L}^{n+1}= & -\left[\left(f_{1}\left(U_{K}^{n+1}\right) k_{2}\left(U_{K}^{n+1}\right)+f_{2}\left(U_{L}^{n+1}\right)\left(k_{1}\left(U_{K}^{n+1}\right)\right)\right] \delta_{K, L}^{n+1}\left(f_{1}(U)\right) \delta_{K, L}^{n+1}\left(p_{c}(U)\right)\right. \\
& -\left[f_{1}\left(U_{K}^{n+1}\right) \delta_{K, L}^{n+1}\left(k_{2}(U)\right)+f_{2}\left(U_{L}^{n+1}\right) \delta_{K, L}^{n+1}\left(k_{1}(U)\right)\right] \delta_{K, L}^{n+1}\left(f_{1}(U)\right) \delta_{K, L}^{n+1}(Q) .
\end{aligned}
$$

Since $\delta_{K, L}^{n+1}(P) \leq 0$ and $\delta_{K, L}^{n+1}(Q) \geq 0$, one of the two terms,

$$
\left[f_{1}\left(U_{K}^{n+1}\right) \delta_{K, L}^{n+1}\left(k_{2}(U)\right)+f_{2}\left(U_{L}^{n+1}\right) \delta_{K, L}^{n+1}\left(k_{1}(U)\right)\right] \delta_{K, L}^{n+1}(P)
$$

or

$$
\left[f_{1}\left(U_{K}^{n+1}\right) \delta_{K, L}^{n+1}\left(k_{2}(U)\right)+f_{2}\left(U_{L}^{n+1}\right) \delta_{K, L}^{n+1}\left(k_{1}(U)\right)\right] \delta_{K, L}^{n+1}(Q)
$$

is non negative. Moreover, since one has:

$$
f_{1}\left(U_{K}^{n+1}\right) k_{2}\left(U_{K}^{n+1}\right)+f_{2}\left(U_{L}^{n+1}\right) k_{1}\left(U_{K}^{n+1}\right) \geq \frac{k_{1}\left(U_{K}^{n+1}\right) k_{2}\left(U_{L}^{n+1}\right)}{k_{1}\left(U_{K}^{n+1}\right)+k_{2}\left(U_{L}^{n+1}\right)}
$$

and

$$
f_{1}\left(U_{K}^{n+1}\right) k_{2}\left(U_{L}^{n+1}\right)+f_{2}\left(U_{L}^{n+1}\right) k_{1}\left(U_{L}^{n+1}\right) \geq \frac{k_{1}\left(U_{K}^{n+1}\right) k_{2}\left(U_{L}^{n+1}\right)}{k_{1}\left(U_{K}^{n+1}\right)+k_{2}\left(U_{L}^{n+1}\right)}
$$

one gets

$$
D_{K, L}^{n+1} \geq \delta_{K, L}^{n+1}\left(f_{1}(U)\right) \int_{U_{K}^{n+1}}^{U_{L}^{n+1}} \frac{k_{1}(a) k_{2}(a)}{k_{1}(a)+k_{2}(a)}\left(-p_{c}{ }^{\prime}(a)\right) \mathrm{d} a=\delta_{K, L}^{n+1}(g(U)) \delta_{K, L}^{n+1}\left(f_{1}(U)\right)
$$

Using $(4.61,4.62)$ and (4.63) yields (4.51).

We now state the following corollary, which is essential for the compactness study.

Corollary 4.1. Under assumptions and notations (2.12)-(2.21) and under the additional Hypotheses (3.30)-(3.31), let $D$ be a finite volume discretization of $\Omega \times(0, T)$ in the sense of Definition 3.3 and let $\left(U_{D}, P_{D}\right)$ be a solution of the finite volume scheme (3.22)-(3.26). Then there exists $C_{9}$, which only 
depends on $k_{1}, k_{2}, p_{c}, \alpha_{1}, \theta_{1}, \alpha_{2}, \theta_{2}, \alpha_{0}, \beta_{0}, \beta_{1}, \Omega, T, u_{0}, \bar{s}, \underline{s}$, and not on $D$, such that the following discrete $L^{2}\left(0, T ; H^{1}(\Omega)\right)$ estimate holds:

$$
\sum_{n=0}^{N} \delta t^{n} \sum_{K \in \mathcal{T}} \sum_{L \in \mathcal{N}_{K}} \tau_{K \mid L}\left(\delta_{K, L}^{n+1}(g(U))\right)^{2} \leq C_{9}
$$

Proof. Since the hypotheses of Corollary 4.1 include that of the technical Proposition 9.1 (given in the appendix), the following inequality holds:

$$
\delta_{K, L}^{n+1}(g(U))^{2} \leq C_{f g} \delta_{K, L}^{n+1}(g(U)) \delta_{K, L}^{n+1}\left(f_{1}(U)\right), \forall(K, L) \in \mathcal{E}, \forall n \in \llbracket 0, N \rrbracket .
$$

It then suffices to apply Proposition 4.4 to conclude (4.64).

\section{Compactness properties}

Using the results of [24], one may deduce from (4.45) the following property:

Corollary 5.1 (Space translates of the pressure). Under assumptions and notations (2.12)-(2.21), let D be a finite volume discretization of $\Omega \times(0, T)$ in the sense of Definition 3.3 and let $\left(U_{D}, P_{D}\right)$ be a solution of (3.22)-(3.26).

Then the value $C_{1}>0$ given by Proposition 4.2, which only depends on $k_{1}, k_{2}, p_{c}, \Omega, T, u_{0}$, $\bar{s}, \underline{s}$, and not on $D$, is such that, for any $\xi \in \mathbb{R}^{d}$, the following inequality holds:

$$
\int_{0}^{T} \int_{\Omega_{\xi}}\left[p_{\mathcal{D}}(x+\xi, t)+p_{g}\left(u_{\mathcal{D}}\right)(x+\xi, t)-p_{\mathcal{D}}(x, t)-p_{g}\left(u_{\mathcal{D}}\right)(x, t)\right]^{2} \mathrm{~d} x \mathrm{~d} t \leq C_{1}|\xi|(2 \operatorname{size}(\mathcal{T})+|\xi|)
$$

where $\Omega_{\xi}=\left\{x \in \mathbb{R}^{d},[x, x+\xi] \subset \Omega\right\}$.

Similarly, we deduce from Corollary 4.1 the following property:

Corollary 5.2 (Space translates of $g(u)$ ). Under assumptions and notations (2.12)-(2.21) and under the additional hypotheses (3.30, 3.31), let $D$ be a finite volume discretization of $\Omega \times(0, T)$ in the sense of Definition 3.3 and let $\left(U_{D}, P_{D}\right)$ be a solution of the finite volume scheme (3.22)-(3.26). Then the value $C_{9}$ given by Corollary 4.1, which only depends on $k_{1}, k_{2}, p_{c}, \alpha_{1}, \theta_{1}, \alpha_{2}, \theta_{2}, \alpha_{0}, \beta_{0}, \beta_{1}, \Omega, T, u_{0}, \bar{s}$, s, and not on $D$, is such that

$$
\int_{0}^{T} \int_{\Omega_{\xi}}\left[g\left(u_{\mathcal{D}}(x+\xi, t)\right)-g\left(u_{\mathcal{D}}(x, t)\right)\right]^{2} \mathrm{~d} x \mathrm{~d} t \leq C_{9}|\xi|(2 \operatorname{size}(\mathcal{T})+|\xi|)
$$

where $\Omega_{\xi}=\left\{x \in \mathbb{R}^{d},[x, x+\xi] \subset \Omega\right\}$.

In the proof of convergence below, an important argument is the strong compactness of the sequence $g\left(u_{D_{n}}\right)$ in $L^{2}(\Omega \times(0, T))$. We already have an estimate of the space translates, we also need an estimate on the time translates of $g\left(u_{D}\right)$ to apply Kolmogorov's theorem. This estimate is given in the following proposition.

Proposition 5.1 (Time translates of $g(u)$ ). Under assumptions and notations (2.12)-(2.21) and under the additional Hypotheses (3.30, 3.31), let $D$ be a finite volume discretization of $\Omega \times(0, T)$ in the sense of Definition 3.3 and let $\left(U_{D}, P_{D}\right)$ be a solution of the finite volume scheme (3.22)-(3.26)

Then there exists $C_{10}$, which only depends on $k_{1}, k_{2}, p_{c}, \alpha_{1}, \theta_{1}, \alpha_{2}, \theta_{2}, \alpha_{0}, \beta_{0}, \beta_{1}, \Omega, T, u_{0}$, $\bar{s}$, and not on $D$, such that, for all $\tau \in(0, T)$, the following discrete estimate holds

$$
\int_{0}^{T-\tau} \int_{\Omega}\left[g\left(u_{\mathcal{D}}(x, t+\tau)\right)-g\left(u_{\mathcal{D}}(x, t)\right)\right]^{2} \mathrm{~d} x \mathrm{~d} t \leq C_{10}(\tau+\operatorname{size}(D)) .
$$


Proof. Some of the techniques used in this proof were introduced in the nonlinear parabolic scalar case in e.g. [24].

Step 1. Proof in the continuous case

We give here the analogue of this proof in the continuous case. The main argument is that the functions $k_{1}(u)(\nabla p)^{2}$ and on $(\nabla g(u))^{2}$ are bounded in $L^{1}(\Omega \times(0, T))$ and that an expression of $u_{t}$ can be drawn from equation (2.5). Using the Fubini-Tonelli theorem, we have:

$$
\int_{\Omega} \int_{0}^{T-\tau}[g(u(x, t+\tau))-g(u(x, t))]^{2} \mathrm{~d} t \mathrm{~d} x=\int_{0}^{T-\tau} A(t) \mathrm{d} t
$$

where $A(t)=\int_{\Omega}\left[g(u(x, t+\tau)-g(u(x, t))]^{2} \mathrm{~d} t \mathrm{~d} x\right.$. Since $g$ is a Lipschitz function with Lipschitz constant $\mathbb{C}_{16}$, one has:

$$
\begin{aligned}
A(t) \leq & C_{16} \int_{\Omega}(g(u(x, t+\tau))-g(u(x, t)))(u(x, t+\tau)-u(x, t)) \mathrm{d} x \\
\leq & C_{16} \int_{\Omega}(g(u(x, t+\tau))-g(u(x, t))) \int_{t}^{t+\tau} u_{t}(x, \theta) \mathrm{d} \theta \mathrm{d} x \\
\leq & C_{16} \int_{\Omega} \int_{t}^{t+\tau}(g(u(x, t+\tau))-g(u(x, t)))\left[\operatorname{div}\left(k_{1}(u) \nabla p\right)(x, \theta)\right. \\
& \left.+f_{1}(c) \bar{s}(x, \theta)-f_{1}(u(x, \theta)) \underline{s}(x, \theta)\right] \mathrm{d} \theta \mathrm{d} x .
\end{aligned}
$$

Now if we develop and integrate by parts in $x$, we obtain:

$$
\begin{aligned}
A(t) \leq & C_{16} \int_{\Omega} \int_{t}^{t+\tau} k_{1}(u(x, \theta)) \nabla p(x, \theta) \nabla g(u(x, t+\tau)) \mathrm{d} \theta \mathrm{d} x \\
& -C_{16} \int_{\Omega} \int_{t}^{t+\tau} k_{1}(u(x, \theta)) \nabla p(x, \theta) \nabla g(u(x, t)) \mathrm{d} \theta \mathrm{d} x \\
& +C_{16} \int_{\Omega} \int_{t}^{t+\tau}\left(f_{1}(c) \bar{s}(x, \theta)-f_{1}(u(x, \theta)) \underline{s}(x, \theta)\right)(g(u(x, t+\tau))-g(u(x, t))) \mathrm{d} \theta \mathrm{d} x .
\end{aligned}
$$

Thanks to the Young inequality, we get:

$$
A(t) \leq \frac{C_{16}}{2}\left(2 A_{1}(t)+A_{2}(t)+A_{3}(t)+2 A_{4}(t)\right)
$$

with

$$
\begin{aligned}
& A_{1}(t)=\int_{t}^{t+\tau} \int_{\Omega} k_{1}(u(x, \theta))(\nabla p(x, \theta))^{2} \mathrm{~d} \theta \mathrm{d} x, \\
& A_{2}(t)=k_{1}(1) \int_{t}^{t+\tau} \int_{\Omega}(\nabla g(u(t)))^{2} \mathrm{~d} \theta \mathrm{d} x, \\
& A_{3}(t)=k_{1}(1) \int_{t}^{t+\tau} \int_{\Omega}(\nabla g(u(t+\tau)))^{2} \mathrm{~d} \theta \mathrm{d} x, \\
& A_{4}(t)=\int_{t}^{t+\tau} \int_{\Omega}\left(\bar{s}(x, \theta)^{2}+\underline{s}(x, \theta)^{2}+C(g)\right) \mathrm{d} \theta \mathrm{d} x .
\end{aligned}
$$


Then using the Fubini theorem, and the bound obtained in the preceding propositions, we obtain that:

$$
\int_{0}^{T} A(t) \leq C \tau
$$

Step 2. Proof in the discrete case

For $t \in[0, T)$, let us denote by $n(t)$ the integer $n \in \llbracket 0, N \rrbracket$ such that $t \in\left[t^{n}, t^{n+1}\right)$. We can write:

$$
\int_{0}^{T-\tau} \int_{\Omega}\left(g\left(u_{\mathcal{D}}(x, t+\tau)\right)-g\left(u_{\mathcal{D}}(x, t)\right)\right)^{2} \mathrm{~d} x \mathrm{~d} t=\int_{0}^{T-\tau} A(t) \mathrm{d} t,
$$

with, for a.e. $t \in(0, T-\tau)$,

$$
A(t)=\sum_{K \in \mathcal{T}} m_{K}\left(g\left(u_{K}^{n(t+\tau)+1}\right)-g\left(u_{K}^{n(t)+1}\right)\right)^{2}
$$

Since $g$ is non decreasing and Lipschitz continuous with constant $C_{16}$ (thanks to Hypotheses (3.30) and (3.31)), one gets:

$$
\begin{aligned}
& A(t) \leq C_{16} \sum_{K \in \mathcal{T}} m_{K}\left(g\left(u_{K}^{n(t+\tau)+1}\right)-g\left(u_{K}^{n(t)+1}\right)\right)\left(u_{K}^{n(t+\tau)+1}-u_{K}^{n(t)+1}\right) \\
& =C_{16} \sum_{K \in \mathcal{T}}\left(g\left(u_{K}^{n(t+\tau)+1}\right)-g\left(u_{K}^{n(t)+1}\right)\right) \sum_{n=n(t)+1}^{n(t+\tau)} m_{K}\left(U_{K}^{n+1}-U_{K}^{n}\right)
\end{aligned}
$$

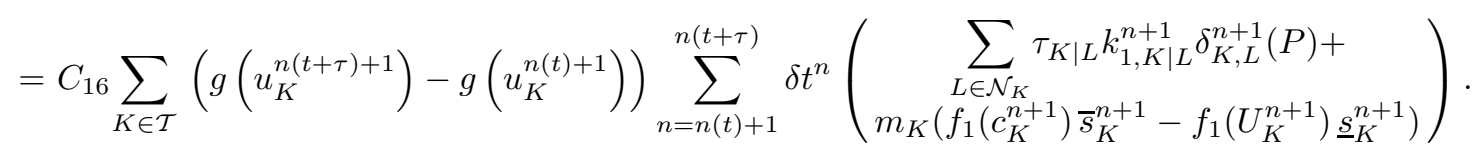

Gathering by edges, we get

$$
\begin{aligned}
A(t) \leq & C_{16} \sum_{n=n(t)+1}^{n(t+\tau)} \delta t^{n} \sum_{K \in \mathcal{T}}\left(\sum_{L \in \mathcal{N}_{K}} \tau_{K \mid L} k_{1, K \mid L}^{n+1} \delta_{K, L}^{n+1}(P)\left(g\left(u_{K}^{n(t+\tau)+1}\right)-g\left(u_{L}^{n(t+\tau)+1}\right)\right)\right) \\
& -C_{16} \sum_{n=n(t)+1}^{n(t+\tau)} \delta t^{n} \sum_{K \in \mathcal{T}}\left(\sum_{L \in \mathcal{N}_{K}} \tau_{K \mid L} k_{1, K \mid L}^{n+1}\left(\delta_{K, L}^{n+1}(P)\left(g\left(u_{K}^{n(t)+1}\right)-g\left(u_{L}^{n(t)+1}\right)\right)\right)\right. \\
& +C_{16} \sum_{n=n(t)+1}^{n(t+\tau)} \delta t^{n} \sum_{K \in \mathcal{T}}\left(m_{K}\left(f_{1}\left(c_{K}^{n+1}\right) \bar{s}_{K}^{n+1}-f_{1}\left(U_{K}^{n+1}\right) \underline{s}_{K}^{n+1}\right)\left(g\left(u_{K}^{n(t+\tau)+1}\right)-g\left(u_{K}^{n(t)+1}\right)\right) .\right.
\end{aligned}
$$

Thanks to the Young inequality, we get

$$
A(t) \leq \frac{C_{16}}{2}\left(2 A_{1}(t)+A_{2}(t)+A_{3}(t)+2 A_{4}(t)\right),
$$


with

$$
\begin{aligned}
& A_{1}(t)=\sum_{n=n(t)+1}^{n(t+\tau)} \delta t^{n} \sum_{K \in \mathcal{T}}\left(\sum_{L \in \mathcal{N}_{K}} \tau_{K \mid L} k_{1, K \mid L}^{n+1}\left(\delta_{K, L}^{n+1}(P)\right)^{2}\right), \\
& A_{2}(t)=k_{1}(1) \sum_{n=n(t)+1}^{n(t+\tau)} \delta t^{n} \sum_{K \in \mathcal{T}}\left(\sum_{L \in \mathcal{N}_{K}} \tau_{K \mid L}\left(g\left(u_{K}^{n(t+\tau)+1}\right)-g\left(u_{L}^{n(t+\tau)+1}\right)\right)^{2}\right), \\
& A_{3}(t)=k_{1}(1) \sum_{n=n(t)+1}^{n(t+\tau)} \delta t^{n} \sum_{K \in \mathcal{T}}\left(\sum_{L \in \mathcal{N}_{K}} \tau_{K \mid L}\left(g\left(u_{K}^{n(t)+1}\right)-g\left(u_{L}^{n(t)+1}\right)\right)^{2}\right), \\
& A_{4}(t)=\sum_{n=n(t)+1}^{n(t+\tau)} \delta t^{n} \sum_{K \in \mathcal{T}} m_{K}\left(\left(\bar{s}_{K}^{n+1}\right)^{2}+\left(\underline{s}_{K}^{n+1}\right)^{2}+C_{11}\right) .
\end{aligned}
$$

Thanks to Lemma 9.3 given in the appendix, we may apply (9.98) to $A_{1}$ with

$$
a^{n+1}=\sum_{K \in \mathcal{T}}\left(\sum_{L \in \mathcal{N}_{K}} \tau_{K \mid L} k_{1, K \mid L}^{n+1}\left(\delta_{K, L}^{n+1}(P)\right)^{2}\right)
$$

and using the estimate (4.43), and again apply (9.98) to $A_{4}$ with

$$
a^{n+1}=\sum_{K \in \mathcal{T}} m_{K}\left(\left(\bar{s}_{K}^{n+1}\right)^{2}+\left(\underline{s}_{K}^{n+1}\right)^{2}+C_{11}\right)
$$

and using Hypothesis (2.17). We then apply (9.99) to $A_{2}$ with $\zeta=\tau$, defining

$$
a^{n}=\sum_{K \in \mathcal{T}}\left(\sum_{L \in \mathcal{N}_{K}} \tau_{K \mid L}\left(g\left(u_{K}^{n}\right)-g\left(u_{L}^{n}\right)\right)^{2}\right)
$$

and using (4.64), and to $A_{3}$, setting $\zeta=0$ and with the same definition for $a^{n}$ and again using (4.64). Thus the proof of (5.67) is complete.

\section{Study of THE LiMit}

Proposition 6.1. Under assumptions and notations (2.12)-(2.21) and under the additional Hypotheses (3.30, 3.31), let $\left(D_{m}\right)_{m \in \mathbb{N}}$ be a sequence of finite volume discretizations of $\Omega \times(0, T)$ in the sense of Definition 3.3 such that $\lim _{m \rightarrow+\infty} \operatorname{size}\left(D_{m}\right)=0$ and such that the $\theta$-regularity property (3.32) is satisfied. Let us again denote by $\left(D_{m}\right)_{m \in \mathbb{N}}$ some subsequence of $\left(D_{m}\right)_{m \in \mathbb{N}}$ such that the sequence of corresponding approximate solutions $\left(u_{D_{m}}, p_{D_{m}}\right)_{m \in \mathbb{N}}$ is such that $u_{D_{m}}$ tends to $u$ in $L^{2}\left(\Omega \times(0, T)\right.$ and $p_{D_{m}}$ tends to $p$ weakly in $L^{2}(\Omega \times(0, T)$, as $m$ tends to infinity, where the functions $u$ and $p$ satisfy:

$$
\begin{aligned}
& u \in L^{\infty}(\Omega \times(0, T)), \text { with } 0 \leq u(x, t) \leq 1 \text { for a.e. }(x, t) \in \Omega \times(0, T), \\
& p \in L^{2}(\Omega \times(0, T)), \\
& p+p_{g}(u) \in L^{2}\left(0, T ; H^{1}(\Omega)\right), \\
& g(u) \in L^{2}\left(0, T ; H^{1}(\Omega)\right) .
\end{aligned}
$$

Then $(u, p)$ is a weak solution of (2.5)-(2.11) in the sense of Definition 2.1. 
Proof. Let $\varphi \in C^{\infty}\left(\mathbb{R}^{d} \times \mathbb{R}\right)$ such that $\varphi(\cdot, T)=0$ and $\nabla \varphi \cdot \mathbf{n}=0$ on $\partial \Omega \times(0, T)$. Thanks to the fact that $\Omega$ is a polygonal subset of $\mathbb{R}^{d}$, the set of such functions $\varphi$ is dense for the norm of $L^{2}\left(0, T ; H^{1}(\Omega)\right)$ in the set of functions $\psi \in C^{\infty}\left(\mathbb{R}^{d} \times \mathbb{R}\right)$ which only satisfy $\psi(\cdot, T)=0$, see [16]. We multiply equations (3.23) and (3.24) by $\varphi\left(x_{K}, t^{n+1}\right)$ and sum over $K \in \mathcal{T}$ and $n \in \llbracket 0, N \rrbracket$. Then there remains to show that the discrete terms converge to the corresponding integrals terms. Using the results of convergence of such terms, which can be found in [24] for example, there only remains to prove that the sequence of discrete terms $\left(C_{m}\right)_{m \in \mathbb{N}}$ defined, for all $m \in \mathbb{N}$, by

$$
C_{m}=-\sum_{n=0}^{N} \delta t^{n} \sum_{K \in \mathcal{T}} \varphi\left(x_{K}, t^{n+1}\right) \sum_{L \in \mathcal{N}_{K}} \tau_{K \mid L} k_{1, K \mid L}^{n+1} \delta_{K, L}^{n+1}(P)=A_{m}+B_{m}
$$

with

$$
A_{m}=-\sum_{n=0}^{N} \delta t^{n} \sum_{K \in \mathcal{T}} \varphi\left(x_{K}, t^{n+1}\right) \sum_{L \in \mathcal{N}_{K}} \tau_{K \mid L} k_{1, K \mid L}^{n+1}\left(\delta_{K, L}^{n+1}(P)+\delta_{K, L}^{n+1}\left(p_{g}(U)\right)\right)
$$

and

$$
B_{m}=-\sum_{n=0}^{N} \delta t^{n} \sum_{K \in \mathcal{T}} \varphi\left(x_{K}, t^{n+1}\right) \sum_{L \in \mathcal{N}_{K}} \tau_{K \mid L} k_{1, K \mid L}^{n+1} \delta_{K, L}^{n+1}\left(p_{g}(U)\right),
$$

converges to $\int_{0}^{T} \int_{\Omega}\left(k_{1}(u(x, t)) \nabla\left(p+p_{g}(u)\right)(x, t)-\nabla g(u(x, t))\right) \cdot \nabla \varphi(x, t) \mathrm{d} x \mathrm{~d} t$ (a similar result then holds for the second equation). This proof can be achieved thanks to two lemmas. Lemma 6.1 applies to the study of the limit of $\left(A_{m}\right)_{m \in \mathbb{N}}$ as $m \rightarrow \infty$ whereas Lemma 6.2 yields the limit of $\left(B_{m}\right)_{m \in \mathbb{N}}$ as $m \rightarrow \infty$.

Lemma 6.1 (Weak-Strong convergence). [34] Let $\Omega$ be a polygonal connected subset of $\mathbb{R}^{d}$, with $d=2$ or 3 and let $T>0$ be given. Let $\left(D_{m}\right)_{m \in \mathbb{N}}$ be a sequence of finite volume discretizations of $\Omega \times(0, T)$ in the sense of Definition 3.3 such that $\lim _{m \rightarrow+\infty} \operatorname{size}\left(D_{m}\right)=0$ and such that the regularity Property (3.32) is satisfied. Let $\left(v_{D_{m}}\right)_{m \in \mathbb{N}} \subset L^{2}(\Omega \times(0, T))$ (resp. $\left(w_{D_{m}}\right)_{m \in \mathbb{N}} \subset L^{2}(\Omega \times(0, T))$ ) be a sequence of piecewise constant functions corresponding (in the sense of Def. 3.4) to a sequence of discrete functions $\left(V_{D_{m}}\right)_{m \in \mathbb{N}}\left(\right.$ resp. $\left.\left(W_{D_{m}}\right)_{m \in \mathbb{N}}\right)$ from $\mathcal{T}_{m} \times \llbracket 0, N_{m}+1 \rrbracket$ to $\mathbb{R}$. Assume that there exists a real value $C_{12}>0$ independent on $m$ verifying

$$
\sum_{n=0}^{N} \delta t^{n} \sum_{K \in \mathcal{T}} \sum_{L \in \mathcal{N}_{K}} \tau_{K \mid L}\left(V_{L}^{n+1}-V_{K}^{n+1}\right)^{2} \leq C_{12},
$$

and that the sequence $\left(v_{D_{m}}\right)_{m \in \mathbb{N}}$ (resp. $\left.\left(w_{D_{m}}\right)_{m \in \mathbb{N}}\right)$ converges to some function $v \in L^{2}(\Omega \times(0, T)$ ) (resp. $w \in L^{2}(\Omega \times(0, T))$ ) weakly (resp. strongly) in $L^{2}(\Omega \times(0, T))$, as $m \rightarrow+\infty$. Let $\varphi \in C^{\infty}\left(\mathbb{R}^{d} \times \mathbb{R}\right)$ such that $\varphi(\cdot, T)=0$ and $\nabla \varphi \cdot \mathbf{n}=0$ on $\partial \Omega \times(0, T)$. For all $m \in \mathbb{N}$, let $A_{m}$ be defined by

$$
A_{m}=-\sum_{n=0}^{N} \delta t^{n} \sum_{K \in \mathcal{T}} \varphi\left(x_{K}, t^{n+1}\right) \sum_{L \in \mathcal{N}_{K}} \tau_{K \mid L} W_{K, L}^{n+1}\left(V_{L}^{n+1}-V_{K}^{n+1}\right),
$$

where, for all $(K, L) \in \mathcal{E}$ and $n \in \llbracket 0, N \rrbracket, W_{K, L}^{n+1}=W_{L, K}^{n+1}$, and $W_{K, L}^{n+1}$ is either equal to $W_{K}^{n+1}$ or to $W_{L}^{n+1}$. Then $v \in L^{2}\left(0, T ; H^{1}(\Omega)\right)$ and

$$
\lim _{m \rightarrow+\infty} A_{m}=\int_{0}^{T} \int_{\Omega} w(x, t) \nabla v(x, t) \nabla \varphi(x, t) \mathrm{d} x \mathrm{~d} t .
$$

We now turn to the statement and the proof of Lemma 6.2.

Lemma 6.2. Under assumptions and notations (2.12)-(2.21) and under the additional Hypotheses (3.30, 3.31), let $\left(D_{m}\right)_{m \in \mathbb{N}}$ be a sequence of finite volume discretizations of $\Omega \times(0, T)$ in the sense 
of Definition 3.3 such that $\lim _{m \rightarrow+\infty} \operatorname{size}\left(D_{m}\right)=0$ and such that the regularity Property (3.32) is satisfied. Let $\left(u_{D_{m}}\right)_{m \in \mathbb{N}} \subset L^{2}(\Omega \times(0, T))$ be a sequence of piecewise constant functions corresponding (in the sense of Def. 3.4) to a sequence of discrete functions $\left(U_{D_{m}}\right)_{m \in \mathbb{N}}$ from $\mathcal{T}_{m} \times \llbracket 0, N_{m}+1 \rrbracket$ to $[0,1]$, such that there exists a real value $C_{13}>0$, verifying, for all $m \in \mathbb{N}$,

$$
\sum_{n=0}^{N} \delta t^{n} \sum_{K \in \mathcal{T}} \sum_{L \in \mathcal{N}_{K}} \tau_{K \mid L} \delta_{K, L}^{n+1}(g(U)) \delta_{K, L}^{n+1}\left(f_{1}(U)\right) \leq C_{13}
$$

Assume that the sequence $\left(u_{D_{m}}\right)_{m \in \mathbb{N}}$ converges in $L^{2}(\Omega \times(0, T))$ to some function $u \in L^{2}(\Omega \times(0, T))$ as $m \rightarrow+\infty$. Let $\varphi \in C^{\infty}\left(\mathbb{R}^{d} \times \mathbb{R}\right)$ such that $\varphi(\cdot, T)=0$ and $\nabla \varphi \cdot \mathbf{n}=0$ on $\partial \Omega \times(0, T)$. Let $\left(B_{m}\right)_{m \in \mathbb{N}}$ be the sequence of real values defined, for all $m \in \mathbb{N}$, by

$$
B_{m}=-\sum_{n=0}^{N} \delta t^{n} \sum_{K \in \mathcal{T}} \varphi\left(x_{K}, t^{n+1}\right) \sum_{L \in \mathcal{N}_{K}} \tau_{K \mid L} k_{1}\left(U_{K, L}^{n+1}\right) \delta_{K, L}^{n+1}\left(p_{g}(U)\right)
$$

where, for all $(K, L) \in \mathcal{E}$ and $n \in \llbracket 0, N \rrbracket, U_{K, L}^{n+1}=U_{L, K}^{n+1}$, and $U_{K, L}^{n+1}$ is either equal to $U_{K}^{n+1}$ or to $U_{L}^{n+1}$. Then the following limit holds:

$$
\lim _{m \rightarrow+\infty} B_{m}=\int_{0}^{T} \int_{\Omega} \nabla g(u)(x, t) \nabla \varphi(x, t) \mathrm{d} x \mathrm{~d} t
$$

Proof. Gathering by edges, the term $B_{m}$ can be rewritten as:

$$
B_{m}=\frac{1}{2} \sum_{n=0}^{N} \delta t^{n} \sum_{(K, L) \in \mathcal{E}} \tau_{K \mid L} k_{1}\left(U_{K, L}^{n+1}\right) \delta_{K, L}^{n+1}\left(p_{g}(U)\right)\left(\varphi\left(x_{L}, t^{n+1}\right)-\varphi\left(x_{K}, t^{n+1}\right)\right) .
$$

Let

$$
B_{1 m}=\frac{1}{2} \sum_{n=0}^{N} \delta t^{n} \sum_{(K, L) \in \mathcal{E}} \tau_{K \mid L} \delta_{K, L}^{n+1}(g(U))\left(\varphi\left(x_{L}, t^{n+1}\right)-\varphi\left(x_{K}, t^{n+1}\right)\right) .
$$

Following the conclusion of Corollary 4.1 which holds under the hypotheses of Lemma 6.2 , one gets $g(u) \in$ $L^{2}\left(0, T ; H^{1}(\Omega)\right)$ and one can apply Lemma 6.1 . This yields:

$$
\lim _{m \rightarrow+\infty} B_{1 m}=\int_{0}^{T} \int_{\Omega} \nabla g(u)(x, t) \nabla \varphi(x, t) \mathrm{d} x \mathrm{~d} t .
$$

We now study $B_{1 m}-B_{m}$. Thanks to the regularity of the test function $\varphi$ and to Properties (2.18) and (2.19) on $k_{1}, k_{2}$ and $p_{c}$, one gets the existence of a real value $C_{14}>0$, which only depends on $\varphi$, such that:

$$
\left|B_{1 m}-B_{m}\right| \leq C_{14} \sum_{n=0}^{N} \delta t^{n} \sum_{(K, L) \in \mathcal{E}} \tau_{K \mid L} d_{K \mid L} A_{K, L}^{n+1},
$$

where $A_{K, L}^{n+1}$ is defined, for all $(K, L) \in \mathcal{E}$ and $n \in \llbracket 0, N \rrbracket$, by:

$$
A_{K, L}^{n+1}=\left(k_{1}\left(U_{K}^{n+1}\right)-k_{1}\left(U_{L}^{n+1}\right)\right)\left(p_{g}\left(U_{L}^{n+1}\right)-p_{g}\left(U_{K}^{n+1}\right)\right)
$$




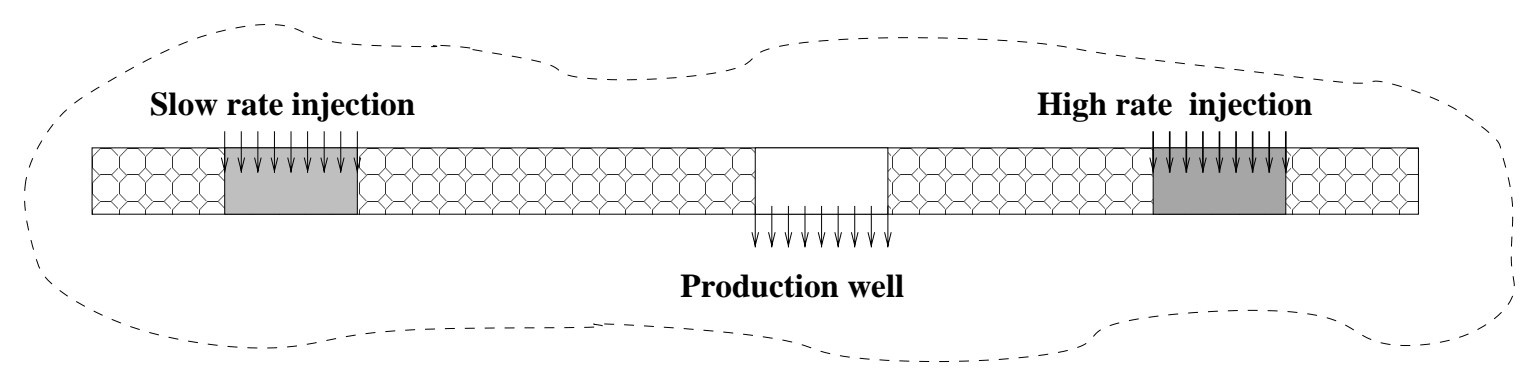

FiguRE 2. Horizontal homogeneous column with injection and production wells.

We now apply the technical Proposition 9.2 (proven in the appendix) with $\delta=d_{K \mid L} / \operatorname{diam}(\Omega), a=U_{K}^{n+1}$ and $b=U_{L}^{n+1}$. This yields:

$$
A_{K, L}^{n+1} \leq C_{p}\left(\frac{\operatorname{diam}(\Omega)}{d_{K \mid L}}\left(f_{1}\left(U_{K}^{n+1}\right)-f_{1}\left(U_{L}^{n+1}\right)\right)\left(g\left(U_{K}^{n+1}\right)-g\left(U_{L}^{n+1}\right)\right)+\frac{d_{K \mid L}}{\operatorname{diam}(\Omega)}\right)\left(\frac{d_{K \mid L}}{\operatorname{diam}(\Omega)}\right)^{\gamma}
$$

which leads, setting $C_{15}=C_{p} \max \left(\operatorname{diam}(\Omega), \frac{1}{\operatorname{diam}(\Omega)}\right) \frac{1}{\operatorname{diam}(\Omega)^{\gamma}}$, to:

$$
A_{K, L}^{n+1} \leq C_{15}\left(\frac{1}{d_{K \mid L}}\left(f_{1}\left(U_{K}^{n+1}\right)-f_{1}\left(U_{L}^{n+1}\right)\right)\left(g\left(U_{K}^{n+1}\right)-g\left(U_{L}^{n+1}\right)\right)+d_{K \mid L}\right)\left(d_{K \mid L}\right)^{\gamma} .
$$

Thus, thanks to Hypothesis (6.68) and thanks to the geometrical property $\frac{1}{2} \sum_{(K, L) \in \mathcal{E}} m_{K \mid L} d_{K \mid L} \leq d \operatorname{meas}(\Omega)$, we get:

$$
\left|B_{1 m}-B_{m}\right| \leq C_{15}\left(C_{13}+d \operatorname{meas}(\Omega)\right) \operatorname{size}(\mathcal{T})^{\gamma}
$$

which shows that $\left|B_{1 m}-B_{m}\right| \rightarrow 0$ as $m \rightarrow \infty$. We thus have completed the proof of Lemma 6.2.

\section{NumERICAL EXPERIMENTS}

In this section, we present some numerical results for the simulation of the flow of two fluids (water and oil) in a porous homogeneous horizontal column. The flow is driven by two injection wells (with different flow rates) located around a unique production well (see Fig. 2).

The purpose of this simple real case is initially to confront the theoretical results concerning the "weakly degenerate" problem studied in this paper to the experiments. In a second step, we shall try to determine the rate of convergence of the scheme. The specific data that we use for this test are the following:

- $\Omega=[0,1]$

- $p_{c}(u)=1-u^{0.7}, k_{1}(u)=u^{2}, k_{2}(u)=2(1-u)$;

- $\bar{s}=10 \chi_{[0.1,0.2]}+30 \chi_{[0.8,0.9]}, \underline{s}=40 \chi_{[0.5,0.6]}$;

- $u_{0}(x)=0, c(x)=0.8$.

We represent in Figure 3, the evolution of the saturation $u$ from $t=0$ to $t=0.5$. Some important numbers like the mean value of the water saturation (equal here to the rate of oil extracted from the reservoir) or the saturation of water at the production well are reported in the table present in Figure 3.

We can observe that the water front $(u>0)$ moves with a finite speed and that the saturation $u$ remains in the interval $[0,0.8]$ related to our choice of $u_{0}$ and $c(x)$. The saturation $u$ is continuous but its gradient can be discontinuous. The production rate increases quickly at the beginning of the process and decreases as the water reaches to the production well. 
water saturation, $\mathrm{t}<0.5$

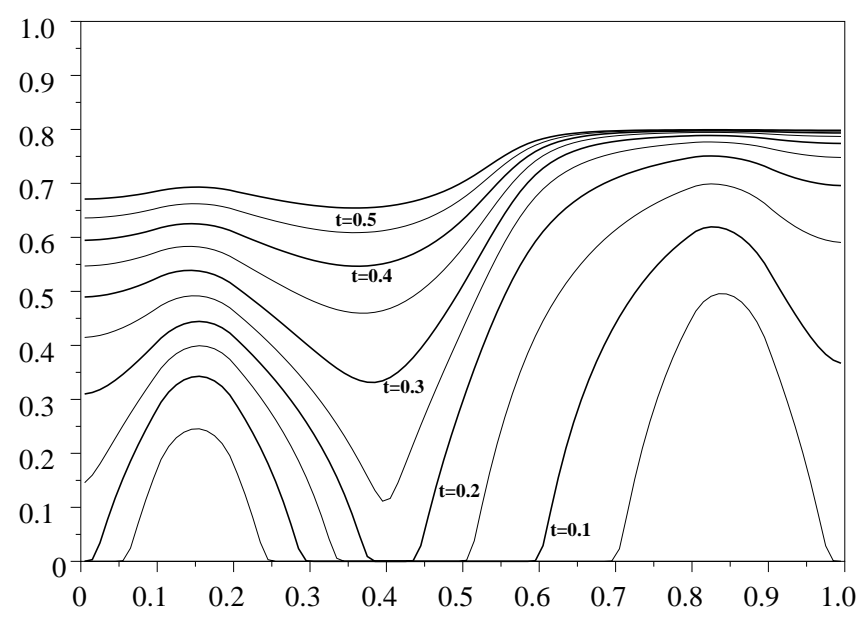

\begin{tabular}{|l|c|c|}
\hline Time & $\left|u_{w}\right|$ & $u_{w}$ (prod) \\
\hline 0.00 & 0.00 & 0.00 \\
0.05 & 0.12 & 0.00 \\
0.10 & 0.24 & 0.00 \\
0.15 & 0.36 & 0.22 \\
0.20 & 0.46 & 0.46 \\
0.25 & 0.53 & 0.56 \\
0.30 & 0.59 & 0.62 \\
0.35 & 0.64 & 0.66 \\
0.40 & 0.68 & 0.70 \\
0.45 & 0.71 & 0.72 \\
0.50 & 0.73 & 0.74 \\
\hline
\end{tabular}

Figure 3. Water saturation evolution from $t=0$ to $t=0.5 .\left|u_{w}\right|:=$ mean value, and $u_{w}(\operatorname{prod}):=$ value at the production well.

TABLE 1. Numerical rates of convergence on the saturation $u$ for the 1D column test, computed from data plotted in Figure 4 and Figure 5.

\begin{tabular}{|l||rrr|rrr|}
\hline \multicolumn{1}{|c||}{} & \multicolumn{3}{c|}{ Exact TVF + Upwind } & \multicolumn{3}{c|}{ PE scheme } \\
\cline { 2 - 7 } & $L^{1}$ & $L^{2}$ & $L^{\infty}$ & $L^{1}$ & $L^{2}$ & $L^{\infty}$ \\
\hline time $: N-2 N$ & 1.001 & 0.998 & 0.996 & 1.001 & 1.000 & 0.976 \\
space $:$ ncv -2 ncv & 1.034 & 0.940 & 0.552 & 0.812 & 0.684 & 0.343 \\
space $:$ ncv - solref & 1.045 & 0.960 & 0.842 & 0.798 & 0.688 & 0.378 \\
\hline
\end{tabular}

In the one-dimensional case, the total velocity flow may be exactly computed, and the problem reduces to a parabolic hyperbolic equation for $u$. This problem, however, is still nonlinear and degenerate, and so no easy analytical solution is known. Hence we shall compute (and this is also the case of the full scheme on the system) an approximate solution on a very fine mesh, and this numerical solution will be used as a reference solution ("solref" in the various tables and figures) to compute the rate of convergence (see Tab. 1). In the tables, we shall denote by "Exact TVF + Upwind" the numerical solution of the parabolic-hyperbolic equations (see [22]), while we shall denote by "PE scheme" the solution of the petroleum engineering scheme.

The two schemes have monotony properties in space and time so that the study of the errors $E_{\text {space }}(n c v)=$ $\left\|u_{N, n c v}-u_{N, 2 n c v}\right\|$ and $E_{\text {time }}(N)=\left\|u_{N, n c v}-u_{2 N, n c v}\right\|$ gives a good indication on the convergence rate in space and time if $N$ is the number of time steps and $n c v$ the number of control volumes. The results are summarized in Figure 4.

The fully implicit upwind scheme used to solve the parabolic degenerate problem is a first order scheme in time and space. Since our problem is only weakly degenerate, the exact solution $u$ is regular (at least continuous), which can explain why the rate of convergence is nearly equal to 1 in the $L^{1}$-norm. For the Petroleum engineering scheme, the analysis is more difficult because it is a coupling of two first order schemes. Nevertheless, the results seem to indicate that the rate of convergence in space is not so far from 0.8 on regular solutions in the $L^{1}$-norm. 

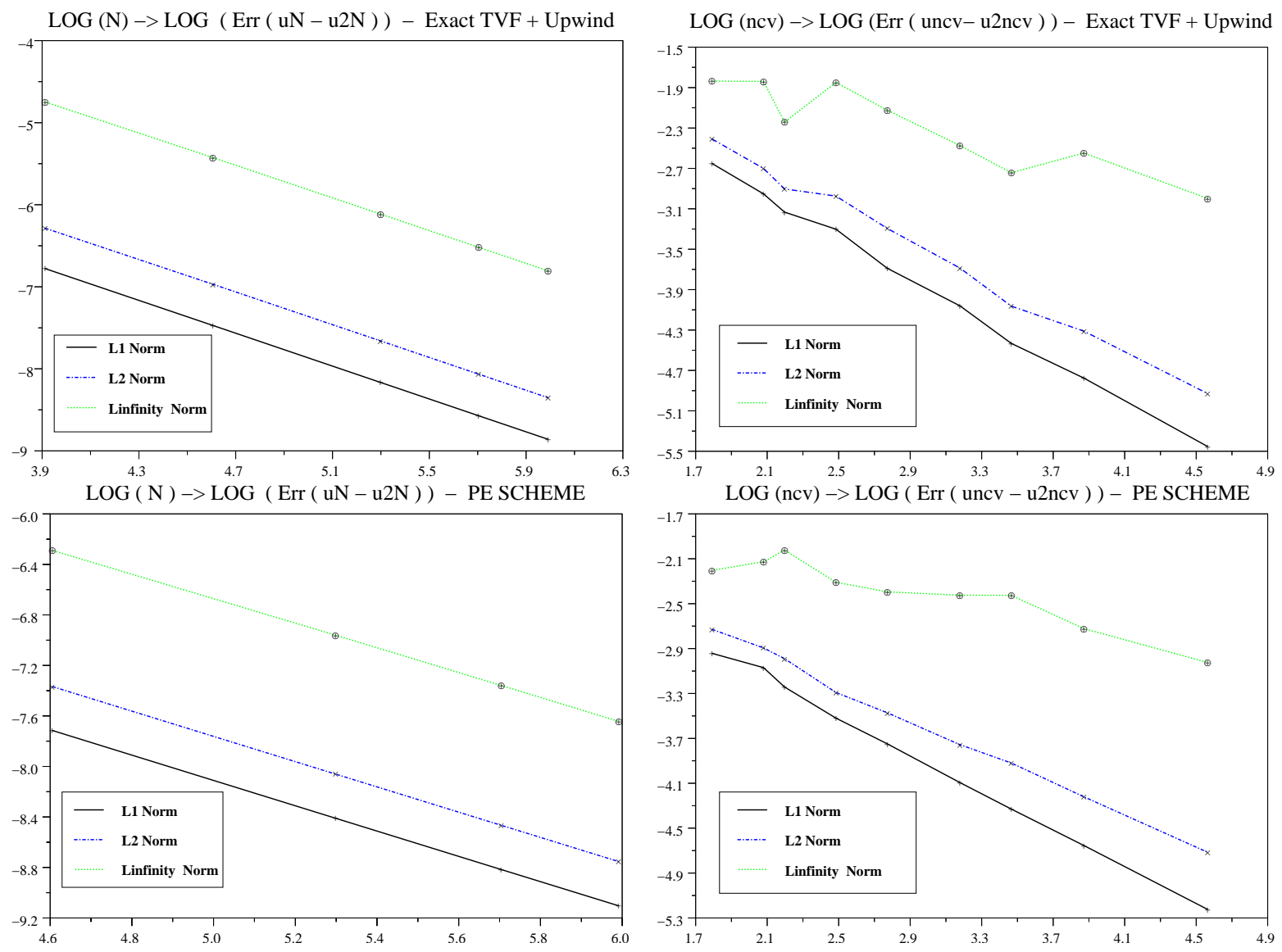

FigurE 4. Log-Log diagrams of the errors $\left\|u_{N, n c v}-u_{2 N, n c v}\right\|$, and $\left\|u_{N, n c v}-u_{N, 2 n c v}\right\|$ for the "Exact TVF + Upwind" method and for the "PE scheme".

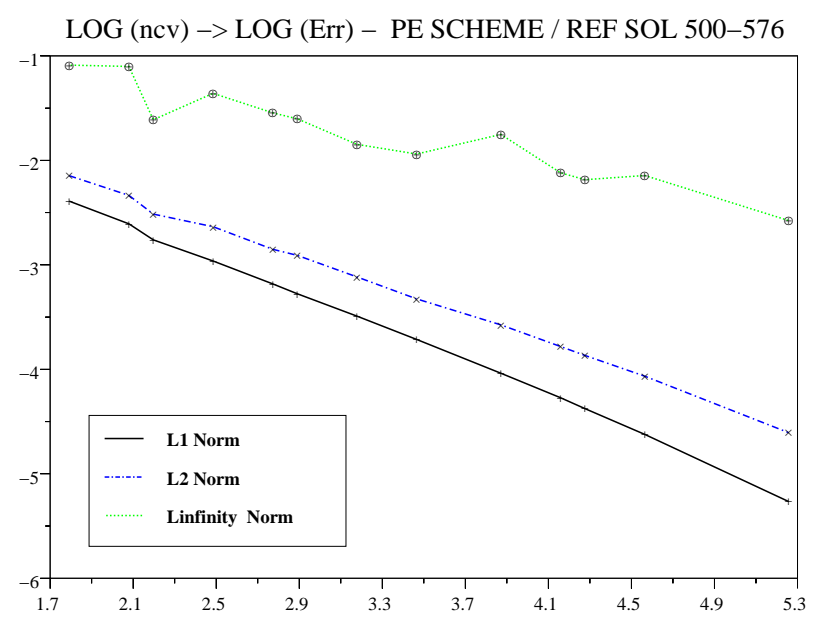

FiguRE 5. Log-Log diagram of the error $\left\|u_{N, n c v}-u_{500,576}^{\mathrm{ref}}\right\|$ for the "PE scheme". 


\section{Concluding Remarks}

In this work, we showed the convergence of the approximate velocities and pressure obtained by a finite volume scheme for the solution of a coupled system of parabolic equations which describes an incompressible two phase flow in a porous media. The question of weaker hypotheses in order to obtain the result of convergence presented here arises. However, the technical assumptions (3.30) and (3.31) seem to cover most of the actual engineering cases. Therefore one can consider other directions for further research; the convergence result should be first extended to more realistic multi-dimensional case in presence of gravity terms. It can secondly be studied in the compressible and compositional cases, but a number of intermediate steps should probably be previously performed.

A first step could be the introduction of a compressible porous medium, that is an approximation of the actual coupling between the flow in the porous media and the mechanical behaviour of the skeleton. In such a case, one could expect that estimates on the time derivative of the pressure might lead to a result of strong convergence for the discrete pressure (in the present paper, this convergence property is only weak, since the time variation of the pressure cannot be controlled in the incompressible case).

A second step would be the introduction of the gravity terms. Some new problems arise, for example in the proof of the discrete maximum principle, or in the proof of the discrete $L^{2}\left(0, T ; H^{1}(\Omega)\right)$ in the multidimensional case (note that new results in this direction have been obtained, in the case of no capillary pressure, in [17]).

\section{Appendix: TECHNICAL PROPOSITIONS}

Proposition 9.1. Under Hypotheses (2.18) and (3.30) on the functions $k_{1}, k_{2}$, and under the Hypotheses (2.19) and (3.31) on the function $p_{c}$, let $f_{1}$ and $g$ be the functions respectively defined by (2.12) and (2.21). Then there exists $C_{f g}>0$, which only depends on $k_{1}, k_{2}, p_{c}, \alpha_{1}, \theta_{1}, \alpha_{2}, \theta_{2}, \alpha_{0}, \beta_{0}$ and $\beta_{1}$, such that:

$$
|g(a)-g(b)| \leq C_{f g}\left|f_{1}(a)-f_{1}(b)\right|, \forall(a, b) \in[0,1]^{2} .
$$

Proof. In the following proof, let us denote by $C_{i}$, for any integer value $i$, various strictly positive real values which only depend on $k_{1}, k_{2}, p_{c}, \alpha_{1}, \theta_{1}, \alpha_{2}, \theta_{2}, \alpha_{0}, \beta_{0}$ and $\beta_{1}$. Thanks to the hypotheses of the proposition, the following inequalities hold:

$$
\begin{gathered}
C_{16} b^{\theta_{1}} \leq k_{1}(b) \leq C_{17} b^{\theta_{1}}, \forall b \in(0,1), \\
C_{18}(1-b)^{\theta_{2}} \leq k_{2}(b) \leq C_{19}(1-b)^{\theta_{2}}, \forall b \in(0,1), \\
C_{20} \leq k_{1}(b)+k_{2}(b) \leq C_{21}, \forall b \in(0,1), \\
C_{22} b^{\theta_{1}-1} \leq f_{1}^{\prime}(b), \quad \text { for a.e. } b \in(0,3 / 4), \\
C_{23}(1-b)^{\theta_{2}-1} \leq f_{1}^{\prime}(b), \text { for a.e. } b \in(1 / 4,1) .
\end{gathered}
$$

Let $(a, b) \in[0,1]^{2}$. We can suppose, without loss of generality, that $0 \leq a \leq b \leq 1$. Let us consider three cases: case $1,(a, b) \in D_{1}=\left\{(a, b) \in[0,1]^{2}, a+1 / 4 \leq b\right\}$, case 2 , where $(a, b) \in D_{2}=\left\{(a, b) \in[0,1]^{2}, a \leq b \leq a+1 / 4\right.$ and $a+b \leq 1\}$, and case 3 where $(a, b) \in D_{3}=\left\{(a, b) \in[0,1]^{2}, a \leq b \leq a+1 / 4\right.$ and $\left.a+b \geq 1\right\}$.

Let us first consider the case $(a, b) \in D_{1}$. Let us define, since $b-a \geq 1 / 4$,

$$
C_{24}=\max _{\left(a^{\prime}, b^{\prime}\right) \in D_{1}}\left(g\left(b^{\prime}\right)-g\left(a^{\prime}\right)\right)
$$


and, since $f_{1}$ is strictly increasing,

$$
C_{25}=\min _{\left(a^{\prime}, b^{\prime}\right) \in D_{1}}\left(f_{1}\left(b^{\prime}\right)-f_{1}\left(a^{\prime}\right)\right)
$$

We thus get

$$
(g(b)-g(a)) \leq \frac{C_{24}}{C_{25}}\left(f_{1}(b)-f_{1}(a)\right) .
$$

We now consider the case $(a, b) \in D_{2}$. We then have $0 \leq a \leq b \leq 3 / 4$ and therefore, thanks to $g^{\prime}(c) \leq$ $C_{26} c^{\theta_{1}+\beta_{0}-1}$, for all $c \in[0,3 / 4]$, we get

$$
(g(b)-g(a)) \leq C_{27}(b-a) b^{\theta_{1}+\beta_{0}-1} \leq C_{27}(b-a) b^{\theta_{1}-1}
$$

and, thanks to $(9.72)$, we get

$$
\left(f_{1}(b)-f_{1}(a)\right) \geq \frac{C_{22}}{\theta_{1}}\left(b^{\theta_{1}}-a^{\theta_{1}}\right) .
$$

Let us consider the subcase $(a, b) \in D_{21}=\left\{\left(a^{\prime}, b^{\prime}\right) \in D_{2}, 2 a^{\prime} \leq b^{\prime}\right\}$. Then

$$
(g(b)-g(a)) \leq C_{27} b^{\theta_{1}}
$$

and

$$
\left(f_{1}(b)-f_{1}(a)\right) \geq \frac{C_{22}}{\theta_{1}}\left(1-\frac{1}{2^{\theta_{1}}}\right) b^{\theta_{1}} .
$$

Thus we get, from (9.75)-(9.76),

$$
(g(b)-g(a)) \leq C_{28}\left(f_{1}(b)-f_{1}(a)\right) .
$$

We now consider the subcase $(a, b) \in D_{22}=\left\{\left(a^{\prime}, b^{\prime}\right) \in D_{2}, 2 a^{\prime} \geq b^{\prime}\right\}$. We then get

$$
(g(b)-g(a)) \leq C_{27} \frac{1}{2^{\theta_{1}-1}}(b-a) a^{\theta_{1}-1}
$$

and

$$
\left(f_{1}(b)-f_{1}(a)\right) \geq \frac{C_{22}}{\theta_{1}}(b-a) a^{\theta_{1}-1} .
$$

This yields

$$
(g(b)-g(a)) \leq C_{29}\left(f_{1}(b)-f_{1}(a)\right) .
$$

Let us now handle the case $(a, b) \in D_{3}$, with a similar method to the case $(a, b) \in D_{2}$, where $1-b$ plays the role of $a$ and $1-a$ that of $b$. Since the inequality $0 \leq 1-b \leq 1-a \leq 3 / 4$ holds, we thus get:

$$
(g(b)-g(a)) \leq C_{30}(b-a)(1-a)^{\theta_{2}-1}
$$

and

$$
\left(f_{1}(b)-f_{1}(a)\right) \geq C_{31}\left((1-a)^{\theta_{2}}-(1-b)^{\theta_{2}}\right) .
$$

Considering the subcases $(a, b) \in D_{31}=\left\{\left(a^{\prime}, b^{\prime}\right) \in D_{3}, 2\left(1-b^{\prime}\right) \leq\left(1-a^{\prime}\right)\right\}$ similarly to the case $(a, b) \in D_{21}$, and the subcase $(a, b) \in D_{32}=\left\{\left(a^{\prime}, b^{\prime}\right) \in D_{3}, 2\left(1-b^{\prime}\right) \geq\left(1-a^{\prime}\right)\right\}$ similarly to the case $(a, b) \in D_{22}$, we obtain the same conclusion. Gathering all the results for $D_{1}, D_{21}, D_{22}, D_{31}$ and $D_{32}$, we thus conclude the proof. 
Proposition 9.2. Under Hypotheses (2.18) and (3.30) on the functions $k_{1}, k_{2}$, and under the Hypotheses (2.19) and (3.31) on the function $p_{c}$, let $p_{g}, f_{1}, g$ be the functions respectively defined by (2.20), (2.12) and (2.21). Then there exists $C_{p}>0$ and $\gamma>0$, which only depend on $k_{1}, k_{2}, p_{c}, \alpha_{1}, \theta_{1}, \alpha_{2}, \theta_{2}, \alpha_{0}, \beta_{0}$ and $\beta_{1}$, such that:

$$
\left(k_{1}(b)-k_{1}(a)\right)\left(p_{g}(a)-p_{g}(b)\right) \leq C_{p} \delta^{\gamma}\left(\frac{1}{\delta}\left(f_{1}(b)-f_{1}(a)\right)(g(b)-g(a))+\delta\right), \forall(a, b) \in[0,1]^{2}, \forall \delta \in(0,1)
$$

Proof. In the following proof, let us again denote by $C_{i}$, for any integer value $i$, various strictly positive real values which only depend on $k_{1}, k_{2}, p_{c}, \alpha_{1}, \theta_{1}, \alpha_{2}, \theta_{2}, \alpha_{0}, \beta_{0}$ and $\beta_{1}$. We apply the same method as that is used in the proof of Proposition 9.1.

Let $(a, b) \in[0,1]^{2}$ and $\delta \in(0,1)$. We again suppose, without loss of generality, that $0 \leq a \leq b \leq 1$. Let us again consider the same three cases: case $1,(a, b) \in D_{1}=\left\{(a, b) \in[0,1]^{2}, a+1 / 4 \leq b\right\}$, case 2 , where $(a, b) \in D_{2}=\left\{(a, b) \in[0,1]^{2}, a \leq b \leq a+1 / 4\right.$ and $\left.a+b \leq 1\right\}$, and case 3 where $(a, b) \in D_{3}=\{(a, b) \in$ $[0,1]^{2}, a \leq b \leq a+1 / 4$ and $\left.a+b \geq 1\right\}$.

Let us first consider the case $(a, b) \in D_{1}$. Let us define, since $b-a \geq 1 / 4$,

$$
C_{32}=\max _{\left(a^{\prime}, b^{\prime}\right) \in D_{1}}\left(k_{1}\left(b^{\prime}\right)-k_{1}\left(a^{\prime}\right)\right)\left(p_{g}\left(a^{\prime}\right)-p_{g}\left(b^{\prime}\right)\right)
$$

and

We thus get

$$
C_{33}=\min _{\left(a^{\prime}, b^{\prime}\right) \in D_{1}}\left(f_{1}\left(b^{\prime}\right)-f_{1}\left(a^{\prime}\right)\right)\left(g\left(b^{\prime}\right)-g\left(a^{\prime}\right)\right)
$$

$$
\left(k_{1}(b)-k_{1}(a)\right)\left(p_{g}(a)-p_{g}(b)\right) \leq \frac{C_{32}}{C_{33}} \delta \frac{1}{\delta}\left(f_{1}(b)-f_{1}(a)\right)(g(b)-g(a)) .
$$

We now consider the case $(a, b) \in D_{2}$. We then have $0 \leq a \leq b \leq 3 / 4$ and therefore, thanks to $-p_{g}{ }^{\prime}(c) \leq C_{34} c^{\beta_{0}}$ for all $c \in[0,3 / 4]$, we obtain

$$
\left(k_{1}(b)-k_{1}(a)\right)\left(p_{g}(a)-p_{g}(b)\right) \leq C_{35}(b-a)^{2} b^{\theta_{1}+\beta_{0}-2}
$$

and, thanks to the inequalities $f_{1}^{\prime}(c) \geq C_{22} c^{\theta_{1}-1}$ and $g^{\prime}(c) \geq C_{36} c^{\theta_{1}+\beta_{0}-1}$ for all $c \in[0,3 / 4]$, we get

$$
\left(f_{1}(b)-f_{1}(a)\right)(g(b)-g(a)) \geq C_{37}\left(b^{\theta_{1}}-a^{\theta_{1}}\right)\left(b^{\theta_{1}+\beta_{0}}-a^{\theta_{1}+\beta_{0}}\right) .
$$

Let us consider the subcase $(a, b) \in D_{21}=\left\{\left(a^{\prime}, b^{\prime}\right) \in D_{2}, 2 a^{\prime} \leq b^{\prime}\right\}$. Then

$$
\left(k_{1}(b)-k_{1}(a)\right)\left(p_{g}(a)-p_{g}(b)\right) \leq C_{35} b^{\theta_{1}+\beta_{0}}
$$

and

$$
\left(f_{1}(b)-f_{1}(a)\right)(g(b)-g(a)) \geq C_{37}\left(1-\frac{1}{2^{\theta_{1}}}\right)\left(1-\frac{1}{2^{\theta_{1}+\beta_{0}}}\right) b^{2 \theta_{1}+\beta_{0}} .
$$

Let $\varepsilon>0$, the value of which will be chosen later on. In the case where $b \leq \varepsilon$, then

$$
\left(k_{1}(b)-k_{1}(a)\right)\left(p_{g}(a)-p_{g}(b)\right) \leq C_{35} \varepsilon^{\theta_{1}+\beta_{0}},
$$

else, from $(9.82,9.83)$,

$$
\left(k_{1}(b)-k_{1}(a)\right)\left(p_{g}(a)-p_{g}(b)\right) \leq C_{38}\left(f_{1}(b)-f_{1}(a)\right)(g(b)-g(a)) \varepsilon^{-\theta_{1}} .
$$


It now suffices to choose $\varepsilon$ such that

$$
\frac{\varepsilon^{\theta_{1}+\beta_{0}}}{\delta}=\frac{\delta}{\varepsilon^{\theta_{1}}}
$$

that is $\varepsilon=\delta^{2 /\left(2 \theta_{1}+\beta_{0}\right)}$, to obtain either, from (9.84),

$$
\left(k_{1}(b)-k_{1}(a)\right)\left(p_{g}(a)-p_{g}(b)\right) \leq C_{35} \delta \delta^{\beta_{0} /\left(2 \theta_{1}+\beta_{0}\right)}
$$

either from $(9.85)$

$$
\left(k_{1}(b)-k_{1}(a)\right)\left(p_{g}(a)-p_{g}(b)\right) \leq C_{38} \frac{1}{\delta}\left(f_{1}(b)-f_{1}(a)\right)(g(b)-g(a)) \delta^{\beta_{0} /\left(2 \theta_{1}+\beta_{0}\right)} .
$$

We now consider the subcase $(a, b) \in D_{22}=\left\{\left(a^{\prime}, b^{\prime}\right) \in D_{2}, 2 a^{\prime} \geq b^{\prime}\right\}$. We then get

$$
\left(k_{1}(b)-k_{1}(a)\right)\left(p_{g}(a)-p_{g}(b)\right) \leq C_{35} 2^{\theta_{1}+\beta_{0}-2}(b-a)^{2} a^{\theta_{1}+\beta_{0}-2}
$$

and

$$
\left(f_{1}(b)-f_{1}(a)\right)(g(b)-g(a)) \geq C_{37}(b-a)^{2} a^{2 \theta_{1}+\beta_{0}-2} .
$$

Let $\varepsilon>0$, the value of which will be chosen later on. In the case where $a \leq \varepsilon$, then

$$
\left(k_{1}(b)-k_{1}(a)\right)\left(p_{g}(a)-p_{g}(b)\right) \leq C_{35} 2^{\theta_{1}+\beta_{0}-2} \varepsilon^{\theta_{1}+\beta_{0}},
$$

else, from $(9.88,9.89)$,

$$
\left(k_{1}(b)-k_{1}(a)\right)\left(p_{g}(a)-p_{g}(b)\right) \leq C_{39}\left(f_{1}(b)-f_{1}(a)\right)(g(b)-g(a)) \varepsilon^{-\theta_{1}} .
$$

It now suffices to again choose $\varepsilon$ such that $\varepsilon=\delta^{2 /\left(2 \theta_{1}+\beta_{0}\right)}$, to obtain either, from (9.90),

$$
\left(k_{1}(b)-k_{1}(a)\right)\left(p_{g}(a)-p_{g}(b)\right) \leq C_{37} \delta \delta^{\beta_{0} /\left(2 \theta_{1}+\beta_{0}\right)}
$$

or, from $(9.91)$

$$
\left(k_{1}(b)-k_{1}(a)\right)\left(p_{g}(a)-p_{g}(b)\right) \leq C_{40} \frac{1}{\delta}\left(f_{1}(b)-f_{1}(a)\right)(g(b)-g(a)) \delta^{\beta_{0} /\left(2 \theta_{1}+\beta_{0}\right)} .
$$

Let us now handle the case $(a, b) \in D_{3}$, with a similar method to the case $(a, b) \in D_{2}$, where $1-b$ plays the role of $a$ and $1-a$ that of $b$. Since the inequality $0 \leq 1-b \leq 1-a \leq 3 / 4$ holds, we thus get

$$
\left(k_{1}(b)-k_{1}(a)\right)\left(p_{g}(a)-p_{g}(b)\right) \leq C_{41}((1-a)-(1-b))^{2}(1-a)^{\theta_{2}+\beta_{1}-1}
$$

and

$$
\left(f_{1}(b)-f_{1}(a)\right)(g(b)-g(a)) \geq C_{42}\left((1-a)^{\theta_{2}}-(1-b)^{\theta_{2}}\right)\left((1-a)^{\theta_{2}+\beta_{1}}-(1-b)^{\theta_{2}+\beta_{1}}\right) .
$$

Considering the subcase $(a, b) \in D_{31}=\left\{\left(a^{\prime}, b^{\prime}\right) \in D_{3}, 2\left(1-b^{\prime}\right) \leq\left(1-a^{\prime}\right)\right\}$ similarly to the case $(a, b) \in D_{21}$, we let $\varepsilon$ be defined by

and we obtain either, if $(1-a) \leq \varepsilon$,

$$
\frac{\varepsilon^{\theta_{2}+\beta_{1}+1}}{\delta}=\frac{\delta}{\varepsilon^{\theta_{2}-1}}
$$

$$
\left(k_{1}(b)-k_{1}(a)\right)\left(p_{g}(a)-p_{g}(b)\right) \leq C_{43} \delta \delta^{\left(\beta_{1}+2\right) /\left(2 \theta_{2}+\beta_{1}\right)},
$$


or

$$
\left(k_{1}(b)-k_{1}(a)\right)\left(p_{g}(a)-p_{g}(b)\right) \leq C_{44} \frac{1}{\delta}\left(f_{1}(b)-f_{1}(a)\right)(g(b)-g(a)) \delta^{\left(\beta_{1}+2\right) /\left(2 \theta_{2}+\beta_{1}\right)}
$$

In the subcase $(a, b) \in D_{32}=\left\{\left(a^{\prime}, b^{\prime}\right) \in D_{3}, 2\left(1-b^{\prime}\right) \geq\left(1-a^{\prime}\right)\right\}$, we obtain, similarly to the case $(a, b) \in D_{22}$ either, if $(1-b) \leq \varepsilon$,

$$
\left(k_{1}(b)-k_{1}(a)\right)\left(p_{g}(a)-p_{g}(b)\right) \leq C_{45} \delta \delta^{\left(\beta_{1}+2\right) /\left(2 \theta_{2}+\beta_{1}\right)}
$$

or

$$
\left(k_{1}(b)-k_{1}(a)\right)\left(p_{g}(a)-p_{g}(b)\right) \leq C_{46} \frac{1}{\delta}\left(f_{1}(b)-f_{1}(a)\right)(g(b)-g(a)) \delta^{\left(\beta_{1}+2\right) /\left(2 \theta_{2}+\beta_{1}\right)} .
$$

Gathering $(9.81,9.86,9.87,9.91,9.93-9.96)$ and (9.97), we thus complete the proof letting $\gamma=\min \left(1, \beta_{0} /\left(2 \theta_{1}+\right.\right.$ $\left.\left.\beta_{0}\right),\left(\beta_{1}+2\right) /\left(2 \theta_{2}+\beta_{1}\right)\right)($ since $\delta \in(0,1))$.

Proposition 9.3. Let $\left(t^{n}\right)_{n \in \mathbb{Z}}$ be a stricly increasing sequence of real values such that $\delta t^{n}:=t^{n+1}-t^{n} i s$ uniformly bounded, $\lim _{n \rightarrow-\infty} t^{n}=-\infty$ and $\lim _{n \rightarrow \infty} t^{n}=\infty$. For all $t \in \mathbb{R}$, we denote by $n(t)$ the element $n \in \mathbb{Z}$ such that $t \in\left[t^{n}, t^{n+1}\right)$. Let $\left(a^{n}\right)_{n \in \mathbb{Z}}$ be a family of non negative real values with a finite number of non zero values. Then

$$
\int_{\mathbb{R}} \sum_{n=n(t)+1}^{n(t+\tau)}\left(\delta t^{n} a^{n+1}\right) \mathrm{d} t=\tau \sum_{n \in \mathbb{Z}}\left(\delta t^{n} a^{n+1}\right), \forall \tau \in(0,+\infty)
$$

and

$$
\int_{\mathbb{R}}\left(\sum_{n=n(t)+1}^{n(t+\tau)} \delta t^{n}\right) a^{n(t+\zeta)+1} \mathrm{~d} t \leq\left(\tau+\max _{n \in \mathbb{Z}} \delta t^{n}\right) \sum_{n \in \mathbb{Z}}\left(\delta t^{n} a^{n+1}\right), \forall \tau \in(0,+\infty), \forall \zeta \in \mathbb{R}
$$

Proof. Let us define the function $\chi(t, n, \tau)$ by $\chi(t, n, \tau)=1$ if $t<t^{n}$ and $t+\tau \geq t^{n}$, else $\chi(t, n, \tau)=0$. We have

$$
\int_{\mathbb{R}} \sum_{n=n(t)+1}^{n(t+\tau)}\left(\delta t^{n} a^{n+1}\right) \mathrm{d} t=\int_{\mathbb{R}} \sum_{n \in \mathbb{Z}}\left(\delta t^{n} a^{n+1} \chi(t, n, \tau)\right) \mathrm{d} t=\sum_{n \in \mathbb{Z}}\left(\delta t^{n} a^{n+1} \int_{\mathbb{R}} \chi(t, n, \tau) \mathrm{d} t\right) .
$$

Since $\int_{\mathbb{R}} \chi(t, n, \tau) d t=\int_{t^{n}-\tau}^{t^{n}} \mathrm{~d} t=\tau$, thus (9.98) is proven.

We now turn to the proof of (9.99). We define the function $\tilde{\chi}(n, t)$ by $\tilde{\chi}(n, t)=1$ if $n(t)=n$, else $\tilde{\chi}(n, t)=0$. We have

which yields

$$
\int_{\mathbb{R}}\left(\sum_{n=n(t)+1}^{n(t+\tau)} \delta t^{n}\right) a^{n(t+\zeta)+1} \mathrm{~d} t=\int_{\mathbb{R}}\left(\sum_{n=n(t)+1}^{n(t+\tau)} \delta t^{n}\right) \sum_{m \in \mathbb{Z}} a^{m+1} \tilde{\chi}(m, t+\zeta) \mathrm{d} t
$$

$$
\int_{\mathbb{R}}\left(\sum_{n=n(t)+1}^{n(t+\tau)} \delta t^{n}\right) a^{n(t+\zeta)+1} \mathrm{~d} t=\sum_{m \in \mathbb{Z}} a^{m+1} \int_{t^{m}-\zeta}^{t^{m+1}-\zeta}\left(\sum_{n=n(t)+1}^{n(t+\tau)} \delta t^{n}\right) \mathrm{d} t
$$


Since we have

we can write from $(9.100)$

$$
\sum_{n=n(t)+1}^{n(t+\tau)} \delta t^{n}=\sum_{n \in \mathbb{Z},}\left(t^{n+1}-t^{n} \leq t+\tau=\tau+\max _{n \in \mathbb{Z}} \delta t^{n}\right.
$$

$$
\int_{\mathbb{R}}\left(\sum_{n=n(t)+1}^{n(t+\tau)} \delta t^{n}\right) a^{n(t+\zeta)+1} \mathrm{~d} t \leq\left(\tau+\max _{n \in \mathbb{Z}} \delta t^{n}\right) \sum_{m \in \mathbb{Z}} a^{m+1} \int_{t^{m}-\zeta}^{t^{m+1}-\zeta} \mathrm{d} t=\left(\tau+\max _{n \in \mathbb{Z}} \delta t^{n}\right) \sum_{m \in \mathbb{Z}} a^{m+1} \delta t^{m}
$$

which is exactly (9.99).

We have the following corollary of Kolmogorov's theorem.

Corollary 9.1 (Consequence of Kolmogorov's theorem). Let $\Omega$ be a bounded open subset of $\mathbb{R}^{d}$, $d \in \mathbb{N}_{*}$, and let $\left(w_{n}\right)_{n \in \mathbb{N}}$ be a sequence of functions such that:

for all $n \in \mathbb{N}, w_{n} \in L^{\infty}(\Omega)$ and there exists a real value $C_{b}>0$ which does not depend on $n$ such that $\left\|w_{n}\right\|_{L^{\infty}(\Omega)} \leq C_{b}$,

there exists a real value $C_{K}>0$ and a sequence of non negative real values $\left(\mu_{n}\right)_{n \in \mathbb{N}}$ verifying $\lim _{n \rightarrow \infty} \mu_{n}=0$ and

$$
\int_{\Omega_{\xi}}\left(w_{n}(x+\xi)-w_{n}(x)\right)^{2} \mathrm{~d} x \leq C_{K}\left(|\xi|+\mu_{n}\right), \forall n \in \mathbb{N}, \forall \xi \in \mathbb{R}^{d}
$$

where, for all $\xi \in \mathbb{R}^{d}$, we set $\Omega_{\xi}=\left\{x \in \mathbb{R}^{d},[x, x+\xi] \subset \Omega\right\}$.

Then there exists a subsequence of $\left(w_{n}\right)_{n \in \mathbb{N}}$, again denoted $\left(w_{n}\right)_{n \in \mathbb{N}}$, and a function $w \in L^{\infty}(\Omega)$ such that $w_{n} \rightarrow w$ in $L^{p}(\Omega)$ as $n \rightarrow \infty$ for all $p \geq 1$.

Proof. We first extend the definition of $w_{n}$ on $\mathbb{R}^{d}$ by the value 0 on $\mathbb{R}^{d} \backslash \Omega$. We then prove that

$$
\lim _{|\xi| \rightarrow 0} \sup _{n \in \mathbb{N}} \int_{\mathbb{R}^{d}}\left(w_{n}(x+\xi)-w_{n}(x)\right)^{2} \mathrm{~d} x=0 .
$$

Indeed, let $\varepsilon>0$. Since $\cup_{\eta>0}\{x \in \Omega, B(x, \eta) \subset \Omega\}=\Omega$, then there exists $\bar{\eta}>0$ such that

$$
\operatorname{meas}(\Omega \backslash\{x \in \Omega, B(x, \bar{\eta}) \subset \Omega\}) \leq \varepsilon .
$$

Thus, for all $\xi \in \mathbb{R}^{d}$ verifying $|\xi| \leq \bar{\eta}$, we have

$$
\{x \in \Omega, B(x, \bar{\eta}) \subset \Omega\} \subset \Omega_{\xi} \cup \Omega_{-\xi} \subset \Omega
$$

and therefore meas $\left(\Omega \backslash\left(\Omega_{\xi} \cup \Omega_{-\xi}\right)\right) \leq \varepsilon$. Let $n_{0} \in \mathbb{N}$ such that, for all $n>n_{0}, \mu_{n} \leq \varepsilon$. Thanks to the theorem of continuity in means, for all $n=0, \ldots, n_{0}$, there exists $\eta_{n}>0$ such that, for all $\xi \in \mathbb{R}^{d}$ verifying $|\xi| \leq \eta_{n}$, we have $\int_{\mathbb{R}^{d}}\left(w_{n}(x+\xi)-w_{n}(x)\right)^{2} \mathrm{~d} x \leq \varepsilon$.

We now take $\xi \in \mathbb{R}^{d}$ verifying $|\xi| \leq \min \left(\bar{\eta},\left(\eta_{n}\right)_{n=0, \ldots, n_{0}}, \varepsilon\right)$. We then get that, for all $n=0, \ldots, n_{0}$, the inequality $\int_{\mathbb{R}^{d}}\left(w_{n}(x+\xi)-w_{n}(x)\right)^{2} \mathrm{~d} x \leq \varepsilon$ holds, and for all $n \in \mathbb{N}$ such that $n>n_{0}$, then

$$
\int_{\mathbb{R}^{d}}\left(w_{n}(x+\xi)-w_{n}(x)\right)^{2} \mathrm{~d} x \leq 4 C_{b}^{2} \varepsilon+\int_{\Omega_{\xi}}\left(w_{n}(x+\xi)-w_{n}(x)\right)^{2} \mathrm{~d} x,
$$

and

$$
\int_{\Omega_{\xi}}\left(w_{n}(x+\xi)-w_{n}(x)\right)^{2} \mathrm{~d} x \leq C_{K}\left(|\xi|+\mu_{n}\right) \leq 2 C_{K} \varepsilon
$$


Gathering the previous results gives (9.101). Then applying Kolmogorov's theorem gives the conclusion of Corollary 9.1.

\section{REFERENCES}

[1] H.W. Alt and E. DiBenedetto, Flow of oil and water through porous media. Astérisque 118 (1984) 89-108. Variational methods for equilibrium problems of fluids, Trento (1983).

[2] H.W. Alt and S. Luckhaus, Quasilinear elliptic-parabolic differential equations. Math. Z. 183 (1983) 311-341.

[3] S.N. Antontsev, A.V. Kazhikhov and V.N. Monakhov, Boundary value problems in mechanics of nonhomogeneous fluids. North-Holland Publishing Co., Amsterdam (1990). Translated from the Russian.

[4] T. Arbogast, M.F. Wheeler and N.-Y. Zhang, A nonlinear mixed finite element method for a degenerate parabolic equation arising in flow in porous media. SIAM J. Numer. Anal. 33 (1996) 1669-1687.

[5] K. Aziz and A. Settari, Petroleum reservoir simulation. Applied Science Publishers, London (1979).

[6] J. Bear, Dynamic of flow in porous media. Dover (1967).

[7] J. Bear, Modeling transport phenomena in porous media, in Environmental studies (Minneapolis, MN, 1992). Springer, New York (1996) 27-63.

[8] Y. Brenier and J. Jaffré, Upstream differencing for multiphase flow in reservoir simulation. SIAM J. Numer. Anal. 28 (1991) 685-696.

[9] J. Carrillo, Entropy solutions for nonlinear degenerate problems. Arch. Rational. Mech. Anal. 147 (1999) $269-361$.

[10] G. Chavent and J. Jaffré, Mathematical models and finite elements for reservoir simulation. Elsevier (1986).

[11] Z. Chen, Degenerate two-phase incompressible flow. I. Existence, uniqueness and regularity of a weak solution. J. Differential Equations 171 (2001) 203-232.

[12] Z. Chen, Degenerate two-phase incompressible flow. II. Regularity, stability and stabilization. J. Differential Equations 186 (2002) 345-376.

[13] Z. Chen and R. Ewing, Mathematical analysis for reservoir models. SIAM J. Math. Anal. 30 (1999) 431-453.

[14] Z. Chen and R.E. Ewing, Degenerate two-phase incompressible flow. III. Sharp error estimates. Numer. Math. 90 (2001) $215-240$.

[15] K. Deimling, Nonlinear functional analysis. Springer-Verlag, Berlin (1985).

[16] J. Droniou, A density result in sobolev spaces. J. Math. Pures Appl. 81 (2002) 697-714.

[17] G. Enchéry, R. Eymard, R. Masson and S. Wolf, Mathematical and numerical study of an industrial scheme for two-phase flows in porous media under gravity. Comput. Methods Appl. Math. 2 (2002) 325-353.

[18] R.E. Ewing and R.F. Heinemann, Mixed finite element approximation of phase velocities in compositional reservoir simulation. R.E. Ewing Ed., Comput. Meth. Appl. Mech. Engrg. 47 (1984) 161-176.

[19] R.E. Ewing and M.F. Wheeler, Galerkin methods for miscible displacement problems with point sources and sinks — unit mobility ratio case, in Mathematical methods in energy research (Laramie, WY, 1982/1983). SIAM, Philadelphia, PA (1984) $40-58$.

[20] R. Eymard and T. Gallouët, Convergence d'un schéma de type éléments finis-volumes finis pour un système formé d'une équation elliptique et d'une équation hyperbolique. RAIRO Modél. Math. Anal. Numér. 27 (1993) 843-861.

[21] R. Eymard, T. Gallouët, M. Ghilani and R. Herbin, Error estimates for the approximate solutions of a nonlinear hyperbolic equation given by finite volume schemes. IMA J. Numer. Anal. 18 (1998) 563-594.

[22] R. Eymard, T. Gallouët, R. Herbin and A. Michel, Convergence. Numer. Math. 92 (2002) 41-82.

[23] R. Eymard, T. Gallouët, D. Hilhorst and Y. Naït Slimane, Finite volumes and nonlinear diffusion equations. RAIRO Modél. Math. Anal. Numér. 32 (1998) 747-761.

[24] R. Eymard, T. Gallouët and R. Herbin, Finite volume methods, in Handbook of numerical analysis, Vol. VII. North-Holland, Amsterdam (2000) 713-1020.

[25] R. Eymard, T. Gallouët and R. Herbin, Error estimate for approximate solutions of a nonlinear convection-diffusion problem. Adv. Differential Equations 7 (2002) 419-440.

[26] P. Fabrie and T. Gallouët, Modeling wells in porous media flow. Math. Models Methods Appl. Sci. 10 (2000) 673-709.

$[27]$ X. Feng, On existence and uniqueness results for a coupled system modeling miscible displacement in porous media. J. Math. Anal. Appl. 194 (1995) 883-910.

[28] P.A. Forsyth, A control volume finite element method for local mesh refinements, in SPE Symposium on Reservoir Simulation. number SPE 18415, Texas: Society of Petroleum Engineers Richardson Ed., Houston, Texas (February 1989) 85-96.

[29] P.A. Forsyth, A control volume finite element approach to NAPL groundwater contamination. SIAM J. Sci. Statist. Comput. 12 (1991) 1029-1057.

[30] Gérard Gagneux and Monique Madaune-Tort, Analyse mathématique de modèles non linéaires de l'ingénierie pétrolière. Springer-Verlag, Berlin (1996). With a preface by Charles-Michel Marle.

[31] R. Helmig, Multiphase Flow and Transport Processes in the Subsurface: A Contribution to the Modeling of Hydrosystems. Springer-Verlag Berlin Heidelberg (1997). P. Schuls (Translator).

[32] D. Kroener and S. Luckhaus, Flow of oil and water in a porous medium. J. Differential Equations 55 (1984) $276-288$. 
[33] S.N. Kružkov and S.M. Sukorjanskiŭ, Boundary value problems for systems of equations of two-phase filtration type; formulation of problems, questions of solvability, justification of approximate methods. Mat. Sb. (N.S.) 104 (1977) 69-88, 175-176.

[34] A. Michel, A finite volume scheme for the simulation of two-phase incompressible flow in porous media. SIAM J. Numer. Anal. 41 (2003) 1301-1317.

[35] A. Michel, Convergence de schémas volumes finis pour des problèmes de convection diffusion non linéaires. Ph.D. thesis, Université de Provence, France (2001).

[36] D.W. Peaceman, Fundamentals of Numerical Reservoir Simulation. Elsevier Scientific Publishing Co (1977)

[37] A. Pfertzel, Sur quelques schémas numériques pour la résolution des écoulements multiphasiques en milieu poreux. Ph.D. thesis, Universités Paris 6, France (1987).

[38] M.H. Vignal, Convergence of a finite volume scheme for an elliptic-hyperbolic system. RAIRO Modél. Math. Anal. Numér. 30 (1996) 841-872.

[39] H. Wang, R.E. Ewing and T.F. Russell, Eulerian-Lagrangian localized adjoint methods for convection-diffusion equations and their convergence analysis. IMA J. Numer. Anal. 15 (1995) 405-459.

To access this journal online:

www.edpsciences.org 